Linguistique, littérature, didactique

151-152 | 2011

Anthropologies de la littérature

\title{
La sociocritique. Définition, histoire, concepts, voies d'avenir
}

\section{Pierre Popovic}

\section{(2) OpenEdition}

1 Journals

\section{Édition électronique}

URL : http://journals.openedition.org/pratiques/1762

DOI : 10.4000/pratiques. 1762

ISSN : 2425-2042

Éditeur

Centre de recherche sur les médiations (CREM)

\section{Édition imprimée}

Date de publication : 15 décembre 2011

Pagination : 7-38

\section{Référence électronique}

Pierre Popovic, «La sociocritique. Définition, histoire, concepts, voies d'avenir », Pratiques [En ligne], 151-152 | 2011, mis en ligne le 13 juin 2014, consulté le 10 décembre 2020. URL : http:// journals.openedition.org/pratiques/1762 ; DOI : https://doi.org/10.4000/pratiques. 1762

(c) Tous droits réservés 


\section{La sociocritique. Définition, histoire, concepts, voies d'avenir}

\section{Pierre Popovic}

Département des littératures de langue française (Université de Montréal) Centre de recherche interuniversitaire en sociocritique des textes (CRIST)

En 1979, dans un texte d'introduction à un collectif disparate néanmoins intitulé Sociocritique, Claude Duchet se voit obligé de redresser la barque. Après un début qui donne le ton : «Commençons par les malentendus. La fortune du mot [sociocritique] est fallacieuse. [...] À trop être étendu, il perd toute pertinence », il ne cesse de rappeler les fondements de la démarche sociocriticienne et finit en ces termes courtois : « Ce recueil pluriel [est] parfois à distance de son objet ${ }^{(1)}$. » En 1989, au terme d'un article de synthèse intitulé " Sociologie de la littérature », Edmond Cros se sent tenu d'apporter cette précision : "Sans doute la sociologie de la littérature et la sociocritique peuvent-elles donner l'impression à première vue qu'elles s'intéressent parfois à des objets identiques mais, au-delà de ces chevauchements apparents, se donnent à voir des préoccupations radicalement opposées ${ }^{(2)}$. » En 2005, dans un entretien avec Ruth Amossy destiné à clore un numéro de revue fort hétérogène lui aussi, Claude Duchet est à nouveau contraint de souligner que « la sociocritique n'est pas une sociologie de la littérature ${ }^{(3)}$ ». En 2008, las de rencontrer la même confusion, des chercheurs fondent le Centre de recherche interuniversitaire en sociocritique des textes (CRIST) et se donnent un texte-manifeste qui s'ouvre sur ce rappel où le mot « sociologie » inclut « sociologie de la littérature » : «La sociocritique n'est ni une discipline ni une théorie. Elle n'est pas non plus une sociologie, encore moins une méthode ${ }^{(4)}$. »

Ces quatre épisodes, choisis parmi bien d'autres, montrent que, de ses débuts aux alentours de 1970 à ses développements les plus contemporains, la sociocritique n'a cessé de marquer sa différence à l'égard de la sociologie de la littérature et de devoir affirmer sa spécificité. Nombre de raisons peuvent être alléguées pour expliquer ce fait.

(1) Claude Duchet (dir.), Sociocritique, Paris, Nathan, 1979, 220 p.

(2) Edmond Cros, "Sociologie de la littérature », dans Marc Angenot, Jean Bessière, Douwe Fokkema, Eva Kushner (dir.), Théorie littéraire, Paris, PUF, 1989, 395 p., pp. 127-149, p. 149.

(3) Ruth Amossy, «Entretien avec Claude Duchet », dans Littérature, n 140 (2005), p. 136.

(4) www.sociocritique-crist.org

(5) Sous l'action de chercheurs usant des moyens classiques de la recherche sociologique et réunis à Bordeaux sous 1'égide de Robert Escarpit, dont le «Que sais-je ? » Sociologie de la littérature, paru en 1958 , avait valeur de certificat de naissance et de reconnaissance académique. 
L'émergence de la sociocritique s'est produite en France alors que la sociologie de la littérature existait «officiellement» depuis une vingtaine d'années ${ }^{(5)}$. L'habitude était prise de mettre tout ce qui se rapprochait du thème "Littérature et société " derrière le même panonceau, d'où la confusion qui s'ensuivit. Nul n'ignore qu'il est difficile de se défaire d'une mauvaise habitude, et nul ne s'étonnera par suite d'apprendre qu'elle resurgit encore quelquefois. Par exemple, dans un ouvrage comme le Dictionnaire du littéraire, la sociocritique n'a droit à aucune notice particulière ; quand son nom apparaît, il ne sert qu'à renvoyer d'une flèche à la notice « sociologie de la littérature ${ }^{(6)}$ ».

En second lieu, le fait que le développement et la diversification de la sociologie de la littérature à partir des années soixante et soixante-dix se sont accomplis en opposition à des institutions universitaires dont les départements de lettres étaient peu réceptifs à tout ce qui fleurait peu ou prou le sociologique, augmenté de la faiblesse en nombre des chercheurs travaillant à ce développement et à cette diversification, a pu justifier d'aucuns de ne pas vouloir établir les différences entre les approches et de ne conserver qu'une seule appellation globale au détriment du travail de distinction et de précision épistémologique nécessaire. L'appellation générale retenue fut celle qui avait déjà pignon sur rue : «sociologie de la littérature », et les esprits pressés ne virent pas ce que la sociocritique apportait d'autre.

Une autre raison vient de ce que certains courants récents de la sociologie de la littérature ont conduit leurs affiliés à intégrer le texte littéraire dans leurs problématiques. Leur postulat est que tout est passible de sociologie, le texte littéraire autant que l'influence des pratiques religieuses sur les mœurs alimentaires et sur les modes de socialisation. Or une vulgate, paresseuse comme elles le sont toutes, s'était bornée à dire de façon simpliste que l'élection du texte au rang d'objet d'étude était la marque distinctive de la sociocritique. C'est la base épistémologique du raisonnement sociocritique ( $c f$. infra), c'est la façon dont le texte est lu et conçu, c'est la manière dont il est relié non pas à des « déterminations sociales objectives » et antérieures (origine, formation, carrière, fortune) mais à des langages conjoncturels qui sont son altérité et qu'il altère toujours, c'est cela qui fait que la sociocritique n'est pas une sociologie et n'est pas de la sociologie de la littérature.

Une dernière cause tient à ce que les sociocriticiens eux-mêmes n'ont pas toujours affirmé leur différence avec la vigueur nécessaire. Le caractère international du développement de la sociocritique, le fait que ses têtes d'affiche évoluèrent en des lieux distants les uns des autres (avant le web et l'ordinateur), l'absence par suite d'un effet de masse critique, un manque de rigueur dans la préparation de plusieurs rencontres et collectifs ${ }^{(7)}$, l'absence sympathique mais dommageable d'une "stratégie d'émergence » et celle, aux côtés des ouvrages de Zima, Cros, Robin et al., d'un fort ouvrage réunissant les propositions fondamentales de Claude Duchet ${ }^{(8)}$, ces circonstances n'ont pas aidé.

Toujours est-il que nous n'en sommes plus là et que ces raisons des confusions passées sont aujourd'hui caduques. L'effet de relatif scandale qui caractérisa vers 1960 l'avènement de recherches sur les relations « du littéraire et du social » est mort de sa plus heureuse mort : des recherches de ce type sont largement présentes dans les programmes et les institutions académiques. L'heure n'est plus à la nécessité de faire corps pour exister ou pour survivre : depuis plus de quarante ans les travaux liés à ce domaine ont été très nombreux et très variés, la moindre bibliographie de thèse sérieuse en témoigne. Le développement international de la sociocritique, s'il pouvait être un handicap vers 1975 ,

(6) Paul Aron et al. (dir.), Le dictionnaire du littéraire, Paris, PUF, 2002, 634 p., p. 557.

(7) Il m'est arrivé comme à bien d'autres de publier des études dans des collectifs ou des actes dont je ne connaissais bien entendu pas par avance la teneur globale.

(8) Ce travail de ressaisie et de publication est par bonheur en cours. 
est aujourd'hui, à l'heure des échanges interuniversitaires, de l'ordinateur et du web, un atout. Nombre de colloques et de rencontres se tinrent soit dans une atmosphère bon-ententiste stérile, sublimée par leurs organisateurs en parangon d'un pluralisme qui n'était dans les faits qu'un indice de civilité, soit dans un climat tendu où les uns se faisaient traiter de « croyants » ou de « naïfs » et les autres de « réducteurs de textes » ou de « laboureurs du champ » : ces rencontres et maints collectifs fluidifiés de la même eau étaient sans doute nécessaires dans une sorte de phase d'exploration, mais ils ont vécu et doivent aujourd'hui laisser place à de vrais débats. Et j'aimerais croire qu'un autre cas de figure, moins excusable, où la confusion entre sociologie de la littérature et sociocritique est délibérée et strictement opportuniste ${ }^{(9)}$, a lui aussi fait son temps.

Car tout travail intellectuel doit porter une attention forte à la définition et au classement des approches cognitives, à la différenciation des objets et des problématiques, aux bases épistémologiques sur lesquelles s'élabore la pensée. C'est sur ce plan et à ce degré d'exigence qu'il faut clairement établir que la sociocritique n'a rien à voir avec la sociologie du livre, rien à voir avec la théorie des champs et la « science des œuvres » proposée par Pierre Bourdieu, rien à voir avec les rabattements de «critique externe » sur du «matériau interne » (ces catégories ne sont pas sociocriticiennes), rien à voir avec les sociologies de la réception. Pour en prendre acte et pour en finir avec tout malentendu, on pointera d'abord les principales avenues de la sociologie de la littérature pour mieux dégager ensuite l'espace ouvert en dehors d'elles par la sociocritique.

\section{La sociologie de la littérature}

Une cartographie des travaux relevant de la sociologie de la littérature fait apparaître plusieurs voies principales de développement ${ }^{(10)}$.

\section{Sociologie de la vie et des pratiques littéraires}

Elle étudie des rapports entre des choses et des choses s'établissant dans l'autour du texte. Fondée sur des enquêtes de terrain et des collectes de données matérielles vérifiables, trouvant ses moyens dans la statistique ou l'entretien, cette sociologie empirique a pour objet le système des relations sociales, des modes de socialisation et des pratiques reliés à l'exercice de la littérature, autrement dit « la vie littéraire » et l'ensemble des conditions de production, de diffusion et de consommation qui sont les siennes. Les travaux de Robert Escarpit et des chercheurs liés à l'École de Bordeaux, portant par exemple sur l'organisation et l'histoire des organisations littéraires (cénacles, académies, maisons d'édition, etc.) ou sur les ressources financières des écrivains, jouèrent en ce domaine un rôle pionnier. Les recherches menées aujourd'hui sur l'histoire de l'édition et les stratégies éditoriales, sur la constitution des réseaux, sur les « sociabilités littéraires », sur la fabrication et les mises en marché du livre relèvent de cette approche ${ }^{(11)}$. Les travaux de

(9) Voir pour exemple de ce confusionnisme délibéré tel collectif de la revue Texte $\left(\mathrm{n}^{\circ} 45 / 46\right)$ basé sur l’idée qu'il est « impossible de définir la sociocritique » quand bien même elle lui sert de titre publicitaire.

(10) Aucune critique des avenues décrites ne sera faite dans cette section et les travaux cités le sont à titre d'exemples. Le but est ici de proposer un tableau aussi synthétique que possible des principaux types de recherches, non d'évaluer les résultats à partir d'un point de vue personnel.

(11) Martine Burgos et al., Sociabilités du livre et communautés de lecteurs : trois études sur la sociabilité du livre, Paris, Bibliothèque publique d'information, Centre Georges Pompidou, 1996, 289 p. ; Philippe Bourdin et Jean-Luc Chappey, Réseaux et sociabilité littéraire en révolution, Clermont-Ferrand, Presses universitaires Blaise-Pascal, 2007, 190 p. ; Jacques Michon et Jean-Yves Mollier (dir.), Les mutations du livre et de l'édition dans le monde du XVIII e siècle à l'an 2000, Sainte-Foy/Paris, Presses de 1'Université Laval/L'Harmattan, 2001, 597 p. ; etc. 
chercheurs comme Roger Chartier, Jacques Michon, Jean-Yves Mollier et du GRÉLQ ${ }^{(12)}$ sur l'édition du livre, les recherches de Nathalie Heinich ${ }^{(13)}$ et de Bernard Lahire ${ }^{(14)}$ sur le métier d'écrivain sont typiques de cette sociologie, à laquelle appartenaient aussi les premières études de Pierre Bourdieu sur Le marché des biens symboliques ${ }^{(15)}$. Dans ce type de recherches où prédominent le regard porté sur les carrières, les réseaux, les mises en marché, les livres et leur vie matérielle, le texte littéraire, s'il est pris en compte, l'est à titre de document, de marchandise ou de complément d'enquête ${ }^{(16)}$.

\section{Analyse de contenus}

Issue de l'ancienne analyse de contenu chère à la sociologie et aux théories de la communication ${ }^{(17)}$, une autre voie s'occupe des valeurs, des idées et des représentations sociales repérables dans les textes de littérature de quelque facture qu'ils soient. Le texte est alors tenu pour un matériau sociologique dans lequel le chercheur cherche des significations localisables, et non des relations de sens toujours beaucoup plus sinueuses et cinétiques. Une partie du travail de Nathalie Heinich va dans ce sens lorsqu'elle puise dans des romans écrits par des femmes des représentations des problèmes identitaires rencontrés par ces dernières dans la vie sociale réelle ${ }^{(18)}$.

\section{Cultural studies}

Nées sous l'égide de l'École de Birmingham et sous l'impulsion de Richard Hoggart et de Raymond Williams ${ }^{(19)}$, les cultural studies ont pour but la critique des formes d'hégémonie culturelle. Elles donnent au concept de culture une portée maximale jusqu'à lui faire désigner tout mode de symbolisation et tout octroi de sens accordé à quelque objet ou fait vital que ce soit. Transdisciplinaires par souci de tenir compte des hybridités et des hétérogénéités inhérentes aux produits culturels, lesquels à leurs yeux sont toujours

(12) Groupe de recherche sur l'édition littéraire au Québec (Université de Sherbrooke).

(13) Être écrivain. Création et identité, Paris, Éditions La Découverte, 2000, 297 p.

(14) La condition littéraire : la double vie des écrivains, Paris, Éditions La Découverte, 2006, 624 p.

(15) Pierre Bourdieu, «Le marché des biens symboliques », dans L'année sociologique, vol. 22, 3 érie, (1971), pp. 49-126. Dans cet article, Bourdieu donne la première mouture du concept de champ littéraire et de la chaîne notionnelle qui lui est reliée. Cette " théorie du champ littéraire » n'est au départ qu'une partie très latérale d'une théorie générale de la domination sociale. La démarche est positiviste (elle cherche des rapports de cause à conséquence, des faits objectifs, et à tirer des lois) et vise en conséquence le général. Les principes méthodologiques sont cependant revus et pensés à partir des sources suivantes : sociologie du goût (Veblen, Schücking), sociologie compréhensive (Weber), structuralisme (en phase d'analyse), psychologie interactionniste (adaptée). La «domination symbolique » est expliquée à l'aide d'un détournement de l'héritage lexical du marxisme (rival conjoncturel qu'il s'agit de combattre en lui prenant ses mots) : lutte, production, biens, producteur, dominé/dominant, capital, etc. En toute logique avec ces bases cognitives, le texte n'est pas étudié comme tel et est considéré comme l'expression de la position des «producteurs dans le champ ». L'histoire du champ est quant à elle vectorialisée par une «tendance à l'autonomisation relative »à l'égard des pouvoirs politiques et religieux.

(16) Il constitue par exemple un passeport social pour son auteur, un moyen de revenu pour un éditeur, etc.

(17) L'ouvrage fondateur de cette analyse est le classique de Bernard Berelson, Content Analysis in Communication Research, Glencoe, The Free Press, 1952, 220 p. Pour une synthèse récente, voir Laurence Bardin, L'analyse de contenu, Paris, PUF, 2003 [11 édition], $291 \mathrm{p}$.

(18) États de femmes. L'identité féminine dans la fiction occidentale, Paris, Gallimard, 1996, 397 p. Ce travail sur la fiction n'est sans doute pas sans trouver des suites dans Mères-filles, une relation à trois (avec Caroline Éliacheff) et dans Les ambivalences de l'émancipation féminine, Paris, Albin Michel, 2002 et 2003,420 p. et 157 p.

(19) On rappellera ces deux ouvrages classiques : Richard Hoggart, La culture du pauvre : étude sur le style de vie des classes populaires en Angleterre, Paris, Minuit, 1970, 422 p. ; Raymond Williams, Culture and Society, 1780-1950, New York, Columbia University Press, 1983, 362 p. 
gros d'un rapport de pouvoir, elles ont connu trois mouvements depuis leur naissance en Angleterre, durant leur développement important aux États-Unis et leur arrivée récente en Europe occidentale continentale. L'adoption du concept de " culture » engendra une première phase dominée par des objets de recherche trouvés dans des corpus, des pratiques et des répertoires déclassés, ceux de la culture populaire, de la culture des jeunes, de la culture des minorités, des "subcultures » (celles entourant le jazz ou le punk, par exemple) : l'analyse des représentations médiatiques en constitua la veine centrale. Influencée par la pensée postcoloniale, par le féminisme, par l'éthique politique, par l'étude des marginalités, ainsi que par le succès états-unien de la French theory (Foucault, Derrida), la seconde phase se caractérise par une focalisation des recherches sur les questions identitaires liées à des groupes et des pratiques comme la sexualité ou la ghettoïsation économique : les gender studies, les gay studies, les queer studies (et al.) en sont des vecteurs majeurs. Enfin, un troisième mouvement, fondé sur une critique des phénomènes reliés à la «mondialisation" (ce terme lui-même est l'objet de nombre de discussions) a dirigé maintes recherches vers les mécanismes de diffusion et de lecture imposés par les industries et les oligopoles culturels contemporains. La littérature et, pire, ses « grands textes » sont pour le moins secondaires dans les études très nombreuses publiées sous le label des cultural studies. En cela fidèle à Hoggart, elles ont cependant pris en compte et soumis à des analyses axiologiques et thématiques des genres et des textes (les romans sentimentaux industriels, les poèmes du jour de l'an, etc.), méprisés par l'histoire littéraire et que les études paralittéraires n'étaient jamais parvenues à considérer autrement que sous l'angle d'une illégitimation phagocytant leur potentiel actif sur le plan des représentations ${ }^{(20)}$.

\section{Sociologie de la création littéraire}

Elle étudie des rapports entre des choses et des mots, c'est-à-dire entre des aspects de la vie sociale des auteurs, le processus de création et son produit (le texte). Deux sous-ensembles peuvent être dégagés dans cette catégorie.

\subsection{Sociopoétique ${ }^{(21)}$, « science des œuvres »}

Elle tire son inspiration de la théorie du champ littéraire de Pierre Bourdieu et, plus particulièrement, de l'inclination nouvelle donnée à cette théorie à partir des Règles de l'artet de leur ambition de fonder une « science des œuvres ». Le " champ littéraire », lieu d'investissement des « habitus » (c'est-à-dire des dispositions acquises sous l'influence du milieu socioculturel et de l'expérience de la vie sociale) et espace des «positions » résultant des relations entre les « agents », les groupes et les institutions, est régi par des rapports de concurrence et de domination; il détermine « arbitrairement » des valeurs et des classements, définit la légitimité des goûts et des pratiques (des façons de parler et d'écrire, par exemple). La logique hiérarchisante de sa structure relationnelle fait qu'il distribue des rôles, impose des conduites et attribue des «positions ». Le but poursuivi est d'étudier le rapport entre la position occupée par l'écrivain dans le champ littéraire et le texte, lequel est le lieu d'une «stratégie » d'accès à la scène symbolique repérable à des

(20) Armand Mattelart et Érik Neveu, Introduction aux Cultural Studies, Paris, La Découverte, 2008, 121 p. ; Hervé Glevarec, Éric Macé et Éric Maigret, Cultural studies. Anthologie, Paris, Armand Colin, 2008, 368 p. ; Chris Baker, Cultural Studies. Theory and Practice, New York, Sage, 2003, 484 p. ; Lawrence Grossberg, Cary Nelson, Paula Treichler (dir.), Cultural Studies, New York, Routledge, 1992, $788 \mathrm{p}$.

(21) Ce terme, proposé par Alain Viala, est sans doute préférable à celui de « science des œuvres », inutilement pompeux. 
éléments particuliers (choix génériques, fabrique de "postures auctoriales », représentations du métier d'écrivain, transposition des conflits esthétiques du moment). La relation entre position et texte en est une d'inférence structurelle causale (le modèle exclut a priori tout volontarisme et toute action pleinement consciente, ce que traduit le choix du mot «agent » par rapport à celui d' " acteur »). Dans les premiers travaux de Bourdieu sur la littérature, le texte est le produit des effets de structure et des interactions et, par suite, l'expression de la position des «producteurs ». Dès lors, la carte des textes est dans un rapport homologique avec le terrain des pratiques de consécration et de légitimation. En un second moment, consécutif à la parution des Règles de l'art ${ }^{(22)}$, Bourdieu, ne renonçant cependant jamais à la catégorie d' "homologie », octroie plus d'autonomie au texte par rapport à son propre modèle initial. Se penchant sur le cas de Flaubert, il accorde au grand écrivain une manière de lucidité par rapport à l'illusio créée par le « jeu » des relations entre les « agents » qui composent le « champ». Plusieurs néobourdieusiens actuels ouvrent davantage cette brèche dans la tendance dominante du modèle au déterminisme et s'efforcent à déglacer une « homologie », parfois trop proche de la spécularité, en accroissant le dynamisme du modèle : écrire et publier un texte équivaut alors à « jouer un coup », à déployer une nouvelle «stratégie » dans un système de positions susceptibles de changements progressifs. L'analyse institutionnelle initiée par Jacques Dubois, les travaux d'Alain Viala, de Denis Saint-Jacques et de Paul Aron, les essais de Jérôme Meizoz (sur les «postures » et « positions ») ou de Fabrice Thumerel émargent entre autres à cette sociopoétique qui comporte toujours une part de sociologie empirique puisqu'elle doit rebâtir l'état du champ à partir de données matérielles objectives et rapporter le texte à cet état.

\subsection{Théorie de la création littéraire}

Un second sous-ensemble, auquel son principal concepteur donne lui-même le nom de " théorie de la création littéraire », s'est dernièrement dégagé. Reconnaissant une dette envers Bourdieu mais critiquant radicalement la portée à ses yeux trop limitée des concepts d'habitus et de champ, Bernard Lahire soutient, dans la suite de ses essais sur $L^{\prime}$ 'homme pluriel ${ }^{(23)}$ et sur La condition sociale des écrivains, que l'examen des seules relations sociales internes à la sphère littéraire est insuffisant pour prendre mesure des « conditions sociales objectives " pesant sur la création littéraire. Prenant l'exemple de Kafka là où Bourdieu avait pris celui de Flaubert, il se donne le projet de savoir «pourquoi Kafka écrit ce qu'il écrit comme il l'écrit » et trouve la réponse dans une « biographie sociologique» qui inventorie les « expériences socialisatrices » de l'auteur. Aux déterminations du champ littéraire se substituent dès lors des «médiations » en cascade. Elles comprennent l'impact de la situation politico-historique, les effets des cadres de vie et des milieux restreints que sont les réseaux familiaux, amicaux et littéraires, ainsi que les habitudes mentales, affectives et comportementales de l'écrivain. Ces expériences socialisatrices mettent à jour une " problématique existentielle », c'est-à-dire des difficultés et des souffrances encourues durant la vie sociale, lesquelles apparaissent sous des formes métaphoriques ou hystérisées dans les textes. À la biographie sectorielle, structuralisée, reformatée par le substrat théorique élaboré par Bourdieu se surimpose dès lors une biographie tirée de textes tenus pour documentaires (journal intime, correspondance, carnets, etc.) et d'essais divers produits par la critique kafkéenne dans une démarche qui a pour aïeul proche Charles-Augustin Sainte-Beuve auquel Lahire ne manque pas de rendre hommage ${ }^{(24)}$.

(22) Les règles de l'art. Genèse et structure du champ littéraire, Paris, Seuil, 1992, $480 \mathrm{p}$.

(23) L'homme pluriel. Les ressorts de l'action, Paris, Nathan, 1998, 271 p.

(24) Franz Kafka. Éléments pour une théorie de la création littéraire, Paris, La Découverte, 2010,632 p. 


\section{Sociologie de la réception des ouvres}

Si la sociologie de la lecture des œuvres se concentre sur des faits objectifs et des données vérifiables tels l'évolution des prix de vente, l'établissement des règles du droit d'auteur, la mise en place des circuits de diffusion, la composition sociale des lectorats (etc.), elle se place alors sous la bannière de la sociologie de la vie et des pratiques littéraires décrite ci-dessus. Elle peut cependant prendre une autre route dès lors qu'elle étudie les réactions que manifestent des publics variés, spécialisés ou non, envers les textes et leur esthétique. Les travaux de Hans-Robert Jauss et de l'École de Constance ont accompagné sur le terrain de l'histoire et de l'étude de l'évolution des normes du goût l'émergence de cette tendance critique dans les années 1980. Pour l' " esthétique de la réception » les textes surviennent dans un «horizon d'attente » que déterminent l'expérience qu'ont les lecteurs du genre de l'œuvre, leur accoutumance à ses thèmes et à sa forme, leur gestion de l'écart entre le langage littéraire proposé et les langages prévalant dans le monde réel. L'œuvre novatrice affiche un écart esthétique décisif par rapport à ces habitudes. Reversée vers le sociologique, cette prise en compte de la lecture peut se donner pour but de dégager des « systèmes de lecture ", composés de façons de lire et d'investissements axiologiques, ainsi que l'ont montré Jacques Leenhardt et Pierre Jòzsa dans une étude comparant des lecteurs français et des lecteurs hongrois ${ }^{(25)}$. Elle peut aussi recourir au concept bourdieusien de «champ littéraire », auquel cas il s'agira de montrer que la lecture est préformatée par l'imposition des normes et des arbitraires culturels, ainsi que par les palmarès et l'idéologie de la littérature produites par les instances de légitimation et de reproduction que sont la critique et l'école. Mais elle peut aussi, quitte à s'appuyer sur la théorie des actes de langage de John L. Austin et à solliciter des études comme celles de Wolfgang Iser sur le lecteur implicite ou d'Umberto Eco sur le lecteur coopérant, se diriger vers une lecture sociopragmatique du texte. Il s'agit alors de chercher comment le texte produit le lecteur dont il a besoin, le faisant complice de son achèvement et de sa possible circulation dans l'espace social. Faisant fond sur une analyse de discours dont il est l'un des spécialistes, Dominique Maingueneau a proposé une réponse personnelle à ce type de questions, associant la prévisibilité de la lecture à des caractéristiques linguistiques et génériques, au partage d'un " pré-dit » (clichés, stéréotypes) et à la « scène énonciative » d'un discours ou d'un texte ${ }^{(26)}$.

Plusieurs travaux de Nathalie Heinich se sont attachés à renouveler cette sociologie de la réception et, même s'ils portent sur l'art contemporain et non sur la littérature, il faut les nommer ici à la fois parce que la littérature est une discipline artistique et en raison des possibilités d'adaptation du modèle proposé à l'étude des textes. Critiquant avec force la logique de la «domination » qui est au centre du modèle bourdieusien, dans lequel elle voit moins une sociologie qu'une idéologie n'osant dire son nom, Heinich tient toute intervention artistique pour une action sociale à part entière dont le sociologue doit mesurer ce qui est mesurable en elle, à savoir ses effets réels sur le monde. Elle écarte logiquement toute herméneutique, puisque les interprétations d'un texte, indéfiniment variables et contradictoires, indiquent qu'il ne peut être intrinsèquement un objet scientifique. Conjuguant en plusieurs livres la présentation d'un modèle théorique et des études de cas, la sociologue montre que l'une des fonctions de l'art contemporain est de conduire le public à se demander ce que c'est que l'art, à se demander si la chose présentée

(25) Jacques Leenhardt et Pierre Jòzsa, avec la collaboration de Martine Burgos, Lire la lecture. Sociologie de la lecture, Paris, Le Sycomore, 1982, 422 p. Voir aussi Martine Burgos, La lecture d'Est en Ouest, Paris, Centre Georges Pompidou, 1993, 119 p.

(26) Wolfgang Iser, L'acte de lecture : théorie de l'effet esthétique, Bruxelles, Pierre Mardaga Éditeur, 1985, 405 p.; Umberto Eco, Lector in fabula, ou La coopération interprétative dans les textes narratifs, Paris, Grasset, 1985, 315 p. ; Dominique Maingueneau, Pragmatique pour le discours littéraire, Paris, Nathan, 2001, 186 p. 
(l' «Emballage du Pont Neuf» en 1985 par Christo à Paris, par exemple) en est ou n'en est pas, à se demander si cela « vaut quelque chose » dans tous les sens possibles de cette expression. L'objet d'analyse consiste dans l'ensemble circonscrit des réactions provoquées par une œuvre auprès de trois groupes de consommateurs : la communauté des artistes, les critiques spécialisés, le grand public. De l'étude comparée de ces réactions parvient à se dégager la forme complexe de la représentation sociale de l'art dans une situation déterminée ${ }^{(27)}$.

\section{La sociocritique : définition}

Par son objet, ses hypothèses heuristiques et sa problématique générale, la sociocritique se distingue radicalement aussi bien de la sociologie empirique que de la sociologie de la littérature. Elle ne s'occupe ni de la mise en marché du texte ou du livre, ni des conditions du processus de création, ni de la biographie de l'auteur, ni de la réception des œuvres littéraires. Elle ne tient pas ces dernières pour un document historique ou sociologique immédiatement lisible comme un exemple ou comme une preuve. Elle n'isole et ne prélève pas des «contenus ». Sa logique épistémologique n'est pas une logique de la preuve, mais une logique de la découverte appliquée aux procès de sens engagés par les textes.

C'est en lui donnant pour objet le texte considéré comme matière langagière, procès esthétique et dispositif sémiotique que Claude Duchet trace dès la fin des années soixante son programme. La « socialité » du texte s'atteint par une lecture interne, immanente, textualiste pour reprendre des mots chargés de mépris par les sociologues : «C'est dans la spécificité esthétique même, la dimension valeur des textes, que la sociocritique s'efforce de lire cette présence des œuvres au monde qu'elle appelle la socialité ${ }^{(28)}$. » Au cours de l'analyse des procédures de mise en texte, la « sociocritique interroge l'implicite, les présupposés, le non dit ou l'impensé, les silences », écrit Duchet, à quoi peuvent s'ajouter les contradictions, les passages énigmatiques, les dérives sémiotiques, les inutilités (personnages surnuméraires, énumérations hasardeuses), l'invention pure et simple (d'une langue par exemple), les relations sémantiques curieuses, les conflits poétiques ou les apories narratives, en clair : tout ce qui relève du sens et non de la signification (étant entendu que le sens est toujours mouvement et la signification arrêt), tout ce qui témoigne d'un déplacement sémiotique productif, tout ce qui porte la trace d'une complexité sémantique et de ce saut véritable dans l'imagination qui caractérise les textes de littérature. Il se comprend à partir de là que la sociocritique ne soit pas une théorie ni une méthode ni une science. Non qu'elle ne mobilise pas des ressources théoriques, non qu'elle ne se pose pas des questions méthodologiques, non qu'elle ne soit animée d'un désir de connaître, mais elle vise nécessairement d'abord le particulier et non le général. L'étude de la mise en texte se nourrit des méthodes de description des textes mises au point dans ce qu'il est convenu d'appeler la « théorie littéraire » et c'est la facture même du texte considéré qui appelle le mode de description ad hoc. Cela signifie que faire de la sociocritique peut se faire en convoquant la simple analyse de texte, la thématique, la narratologie, la rhétorique, la poétique, l'analyse de discours, la linguistique textuelle, etc. et ce qu'il faudra, y compris, par exemple, la praxématique ou la psychanalyse, mais cette convocation en sera une de moyens, non d'une fin. Il revient au sociocriticien de choisir le mode d'analyse et de description approprié ; il ira aussi vers ses penchants personnels et

(27) Nathalie Heinich, Le triple jeu de l'art contemporain : sociologie des arts plastiques, Paris, Minuit, 380 p. et Ce que l'art fait à la sociologie, Paris, Minuit, 1998, 90 p.

(28) Claude Duchet, "Introductions. Positions et perspectives », dans Claude Duchet, Bernard Merigot et Amiel van Teslaar (dir.), Sociocritique, Paris, Nathan, 1979, 220 p., pp. 3-8, p. 4. 
sera prié d'avoir de l'imagination. Quand bien même elles paraîtraient aller de soi, ces précisions ne sont pas inutiles en ces temps où l'enseignement des lettres lui-même tend à réduire à une peau de chagrin la liberté interprétative des jeunes lecteurs ${ }^{(29)}$. Elles posent que la sociocritique est une lecture active des textes sans considération autre que très secondaire des processus de création et de réception, lesquels sont axiomatiquement hors de sa portée. Et elles soulignent pourquoi la sociocritique ne peut pas être une sociologie de la littérature : en effet, raccorder une construction herméneutique dont le critique/lecteur est responsable à des faits matériels concernant la carrière ou l'existence de l'auteur, que ceux-ci soient formatés en "position dans le champ » ou indexés dans une «biographie sociologique », c'est ou bien rejoindre une tradition occultiste qui voyait partout la patte d'une conspiration de la matière ou bien chérir sans le savoir le fétichisme épistémologique.

S'il est ainsi un " textualisme » sociocriticien parfaitement assumé, il faut bien voir que la finalité de l'exercice n'est pas celle poursuivie par les lectures formalistes de jadis et naguère. En sociocritique, l'examen de la mise en forme n'a de sens que par l'éversion du texte vers ses altérités constitutives, c'est-à-dire vers les mots, les langages, les discours, les répertoires de signes qu'il a intégrés, qu'il corrèle les uns aux autres de façon étonnante et problématique, et qu'il transforme grâce à la distance sémiotique qu'il gagne sur eux par divers moyens scripturaux qu'il s'agit justement de faire apparaître et d'analyser. L'étude de «l'organisation interne des textes, leurs systèmes de fonctionnement, leurs réseaux de sens, leurs tensions » s'effectue donc en corrélation avec l'examen de «la rencontre en eux de discours et de savoirs hétérogènes ${ }^{(30)}$ ». L'observation du répertoire des savoirs et des discours pris en écharpe par l'écriture conduit à montrer comment ils sont reliés à des éléments a priori sans rapport avec eux ${ }^{(31)}$, reconfigurés, transformés, ouverts à la polysémie par elle. La socialité et l'historicité du texte se lisent dans ce travail où la reprise compte moins que la rupture opérée, l'emprunt moins que sa déformation active et que le potentiel d'innovation inhérent à cette dernière. Le sociocriticien pose donc qu'il existe telle chose qu'une singularité sociosémiotique du texte. Celle-ci n'est ni un absolu ni une essence, puisqu'elle résulte d'une action, d'une intervention sur et dans la semiosis sociale qui, une fois accomplie, relance vers celle-ci de nouvelles possibilités de sens.

Ce qui spécifie la sociocritique, c'est l'ensemble du geste critique qui vient d'être décrit. Il comprend trois étapes entre lesquelles doivent s'établir en cours de lecture des allées et venues permanentes : 1 . Analyse interne de la mise en texte selon les principes énoncés ci-dessus ; 2 . Éversion inductive du texte vers ses altérités langagières constitutives, c'est-à-dire vers les répertoires lexicaux, les langages sociaux, les discours, les représentations, les images éventuelles qu'il mobilise et travaille en " son dedans », autrement dit : vers la semiosis sociale ${ }^{(32)}$ environnante prise en partie ou saisie en sa totalité ; 3. Étude de la relation bidirectionnelle (en aller-retour) unissant le texte à la semiosis sociale ou à la partie de celle-ci considérée.

Seuls relèvent de la sociocritique les travaux dont la démarche épouse ce geste critique, lequel profile un cadre heuristique où peuvent s'inscrire un nombre pratiquement infini de problématisations et de façons de travailler co-intelligibles. Identité du geste critique et variété méthodologique des approches conduisent à penser la sociocritique d'une manière perspectiviste et à en donner la définition générale suivante :

(29) Voir sur ce point l'essai bienvenu d'Yves Citton : Lire, interpréter, actualiser, Paris, Éditions d'Amsterdam, 2007, $363 \mathrm{p}$

(30) Claude Duchet, "Introductions. Positions et perspectives », dans Claude Duchet, Bernard Merigot et Amiel van Teslaar (dir.), Sociocritique, Paris, Nathan, 1979, 220 p., pp. 3-8, p. 4.

(31) Le discours scientifique et la libido du narrateur dans les romans de Michel Houellebecq par exemple.

(32) C'est-à-dire sur la façon dont une société se représente ce qu'elle est et son devenir par tous les dispositifs sémiotiques de nature langagière dont elle dispose. 
La sociocritique n'est ni une discipline ni une théorie. Elle n'est pas non plus une sociologie, de quelque sorte qu'elle soit, encore moins une méthode. Elle constitue une perspective. À ce titre, elle pose comme principe fondateur une proposition heuristique générale de laquelle peuvent dériver de nombreuses problématiques individuellement cohérentes et mutuellement compatibles.

Cette proposition se présente comme suit :

Le but de la sociocritique est de dégager la socialité des textes. Celle-ci est analysable dans les caractéristiques de leurs mises en forme, lesquelles se comprennent rapportées à la semiosis sociale environnante prise en partie ou dans sa totalité. L'étude de ce rapport de commutation sémiotique permet d'expliquer la forme-sens (thématisations, contradictions, apories, dérives sémantiques, polysémie, etc.) des textes, d'évaluer et de mettre en valeur leur historicité, leur portée critique et leur capacité d'invention à l'égard du monde social. Analyser, comprendre, expliquer, évaluer, ce sont là les quatre temps d'une herméneutique. C'est pourquoi la sociocritique - qui s'appellerait tout aussi bien "sociosémiotique "-peut se définir de manière concise comme une herméneutique sociale des textes.

\section{Historique}

La sociocritique a quelque quarante ans d'existence. La définition qui vient d'en être donnée rend compte de la confluence des travaux qui respectent le geste lecteur qui la fonde. Elle ne s'est évidemment pas développée comme un corps doctrinaire, puisqu'elle n'en est pas un, ni de façon monovectorielle et sectaire, puisque sa perspective ouvre un espace de pensée et n'impose aucun mode d'emploi préconstruit. Depuis le moment où Claude Duchet en formalisa les propositions initiales et les condensa dans un article fondateur $^{(33)}$, elle a essaimé au niveau international, a pris racine ici et là, non sans quelques malentendus occasionnels comme cela a été rappelé ci-dessus, a élu demeure dans des groupes, des "écoles » et trouvé palace dans des œuvres solitaires fortes. Du côté des groupes et des filières, il faut citer les suivants. L'école de Paris VIII a rassemblé autour de Duchet et au fil des ans des chercheurs comme Isabelle Tournier, Stéphane Vachon, Patrick Maurus, Xavier Bourdenet, In-Kyoung Kim, Roseline Tremblay. Contemporaines de celles de Claude Duchet les propositions d'Edmond Cros servirent de base à l'Institut de sociocritique qu'il fonda à l'Université Paul-Valéry de Montpellier. Inspirée par des chercheurs comme André Belleau, Gilles Marcotte, Régine Robin, Marc Angenot, l'École de Montréal a trouvé naguère un lieu de débat et de développement dans le Centre interuniversitaire d'analyse du discours et de sociocritique des textes (CIADEST) et aujourd'hui dans le Centre de recherche interuniversitaire en sociocritique des textes (CRIST), lequel s'offre pour un lieu international ouvert à tous les sociocriticiens. Au nombre des œuvres solitaires fortes précitées figurent celles de Pierre V. Zima et d'Henri Mitterand sur le roman, de Philippe Hamon transformant radicalement l'analyse idéologique par rapport à ce qu'elle avait été à l'ombre du marxisme facultaire des années cinquante et soixante.

Une bibliographie critique de la sociocritique, prolongeant, actualisant et augmentant la «Bibliographie historique » établie par Stéphane Vachon et Isabelle Tournier ${ }^{(34)}$, est à

(33) Claude Duchet, «Pour une sociocritique ou variations sur un incipit », dans Littérature, $\mathrm{n}^{\circ} 1,1971$, pp. $5-14$.

(34) Stéphane Vachon et Isabelle Tournier, "Sociocritique. Bibliographie historique », dans Jacques Neefs et Marie-Claire Ropars (dir.), La politique du texte. Enjeux sociocritiques, Lille, Presses universitaires de Lille, 1992, 277 p., pp. 231-277. Outre la "Bibliographie des écrits de Claude Duchet » (pp. 231-248), la partie spécifiquement consacrée à la sociocritique couvre les pages 253 à 262 (suivent des pages indexant des titres rapportés à la sociologie de la littérature). 
faire. Elle aura aujourd'hui les moyens technologiques nécessaires pour être à la fois aussi complète et sélective que possible. Elle tiendra compte des nombreux travaux dont la démarche correspond au geste critique de la sociocritique décrit ci-dessus sans que cette dernière soit nécessairement nommée, à l'exemple de ceux de Dolf Oehler, Ross Chambers ou Juan Rigoli ${ }^{(35)}$. Elle signalera aussi les parties de livres et de collectifs qui la concernent, même si le volume au complet a d'autres visées.

Une histoire précise de la sociocritique n'est pas moins à faire et demanderait une bonne thèse de doctorat. Elle prendra en compte les penseurs, de quelque aire géographique qu'ils soient, qui, avant Duchet, Zima et Cros, s'efforcèrent de saisir la particularité d'une écriture en décalage des configurations sémiotiques circulant dans l'espace social, à l'exemple de Walter Benjamin lisant Baudelaire ${ }^{(36)}$ ou d'Erich Köhler ${ }^{(37)}$ analysant les présences du hasard dans le roman occidental. Si elle ne négligera pas les alluvions, elle se méfiera de tout messianisme rétroactif ${ }^{(38)}$ et notera les adossements philosophiques et théoriques successifs des chercheurs ${ }^{(39)}$. Le tournant des années $60 / 70$ sera l'objet d'une attention particulière. Dans ses textes fondateurs, Duchet situe alors la sociocritique dans un « entre-deux », lequel sépare l'avant ( sociologie de la création ») et l'après ("sociologie de la lecture»). Vu d'aujourd'hui le paysage théorique paraît avoir été plus chargé et plus effervescent que cela. Loin de la théorie de la domination symbolique que lance Bourdieu, loin de la tradition anglo-saxonne des études de carrière et de constitutions de groupes que vient de relayer en France la sociologie empirique de l'École de Bordeaux, la sociocritique a cinq assistants majeurs autour de ses fonts baptismaux : tout ce qui, depuis les formalistes russes jusqu'aux sémioticiens, a nourri l'étude immanente des textes ; le structuralisme génétique de Lucien Goldmann ; la sémantique historique étudiant le devenir en longue durée de la signification des mots et leur circulation dans la parole publique ${ }^{(40)} ;$ 1'analyse de discours ${ }^{(41)}$; 1'analyse idéologique. Issue du marxisme, redorée par Louis Althusser, inséparable du jugement de « fausse conscience », cette dernière sombre peu à peu dans un mécanisme et un manichéisme stériles. Il suffit de replonger dans des articles de l'époque pour apercevoir que tout et n'importe quoi sont rapportés à une "idéologie dominante » qui a d'autant plus bon dos qu'elle empêcherait ou retarderait la marche inévitable de la lutte des classes vers l'avenir radieux. Tant Duchet et Cros que les chercheurs des années 1980 travaillent à sortir de ce manichéisme et à penser l'idéologique comme le résultat d'un effet produit par des discours et par des textes précis.

Cette histoire de la sociocritique montrera ensuite l'importance qu'ont eue les concepts d'intertextualité et d'interdiscursivité à la fois pour sortir de l'impasse où menaçait de stagner l'analyse idéologique et pour garantir la possibilité d'une lecture sociale des textes soucieuse des conséquences sémantiques de leur mise en forme et de leur intervention sur les langages. Alors que les grands essais de Theodor Adorno, de Walter Benjamin

(35) Dolf Oehler, Le spleen contre l'oubli. Juin 1848. Baudelaire, Flaubert, Heine, Herzen, Paris, Payot, 1996, 465 p. ; Ross Chambers, Loiterature, Lincoln, University of Nebraska Press, 1999, 311 p. ; Rigoli, Juan. Lire le délire. Aliénisme, rhétorique et littérature en France au XIX siècle. Paris, Fayard, 2001, $649 \mathrm{p}$.

(36) Walter Benjamin, Charles Baudelaire : un poète lyrique à l'apogée du capitalisme. Traduit de l'allemand et préfacé par Jean Lacoste d'après l'édition originale établie par Rolf Tiedemann, Paris, Petite Bibliothèque Payot, 1982, 286 p.

(37) Erich Köhler, Le hasard en littérature. Le possible et la nécessité, Paris, Klincsieck, 1987, 128 p.

(38) Par conséquent elle défera de curieuses trames (faussement) historiques alléguant que Lukàcs, Adorno, Mukàrovsky et cent autres auraient été dans l'erreur, faute d'avoir reçu la révélation apportée par tel sociologue récent.

(39) Ainsi prendra-t-on en compte les pages où Germaine de Staël, dès lors fidèle à Montesquieu, rapporte la littérature d'un pays non à son organisation, mais à l'esprit de ses institutions.

(40) Jean Dubois, plus tard Maurice Tournier.

(41) Michel Pêcheux, moins clairement quelques travaux de Michel Foucault. 
et de l'école de Francfort, ainsi que ceux de Mikhaïl Bakhtine sont alors fort peu connus en France et à peine (très partiellement) traduits ${ }^{(42)}$, ils sont au centre de 1'approche sociocriticienne grâce au travail de Pierre V. Zima, heureusement rapidement diffusé (en plusieurs langues) dès les années 1980. L'héritage bakhtinien est ensuite au cœur du développement de l'École de Montréal et de ses incarnations successives dans divers travaux et groupes : les travaux de Marc Angenot sur le concept de discours social, l'articulation entre texte et discours social sont alors au centre des débats. Il en sera question dans les pages qui suivent, ainsi que de « la sociocritique qui se fait».

\section{Les travaux et les jours : auteurs, recherches et concepts}

Les problématiques conçues dans la perspective de la sociocritique se différencient les unes des autres de plusieurs façons. La conformation du texte considéré appelle premièrement une approche ad hoc, respectueuse des choix esthétiques qui ont présidé à sa mise en place. L'incipit des Bienveillantes (« Frères humains, laissez-moi vous raconter comment ça s'est passé ${ }^{(43)}$.») et un poème exploréen de Claude Gauvreau (« Thathamausauskayakutès ${ }^{(44)}$ ») ne peuvent d'évidence s'entreprendre avec les mêmes moyens. Leur différenciation dépend en deuxième lieu de la saisie de la semiosis sociale alentours, aussi bien de son envergure (saisie partielle ou totalisante) que de sa nature. Troisièmement elle varie en fonction des ressources théoriques secondaires mobilisées. À cela s'ajoutent des qualités particulières du sociocriticien, dont sa façon propre d'écrire et de conduire sa prose. C'est là une chose qui ne se dit pas très souvent dans le domaine de la recherche académique, et c'est une erreur : il n'y a pas de sociocritique qui vaille sans une écriture suffisamment alerte, dynamique et inventive pour être capable de rendre au mieux les mouvements de sens du ou des texte(s) étudié(s).

Le concept de sociogramme a des accointances avec la sémantique historique. Claude Duchet le définit comme un « ensemble flou, instable, conflictuel, de représentations partielles, aléatoires, en interaction les unes avec les autres, gravitant autour d'un noyau lui-même conflictuel ${ }^{(45)} \gg$. Cette définition, souvent glosée, appelle pour être comprise les notions corrélées d'information, d'indice et de valeur, ainsi que celle de cotexte. Le réel (le référent social comme tel) reste en dehors de la prise, et il est toujours déjà dit sous la forme d'une masse d'informations brutes composant une première rumeur touffue, faite de faits allégués, de toponymes, d'événements, d'évidences, de tuf mémoriel et de premières configurations discursives imprécises. Le texte, quant à lui, intègre et désigne un cotexte dont il est partiellement fait et sur lequel il agit. Ce cotexte ${ }^{(46)}$ est composé de ces informations référentielles déjà sémiotisées par des discours et traduites en « indices » par cette première sémiotisation ${ }^{(47)}$. La textualisation ou mise en texte consiste à la fois dans cette reprise indicielle et dans la transformation par l'écriture de ces « indices » en « valeurs », c'est-à-dire en unités qui, via le travail d'écriture et d'esthétisation, prennent une charge sémantique discrète (elles se différencient les unes des autres), pleine

(42) Le dialogisme bakhtinien, le concept d'intertexte, les écrits du «Cercle de Bakhtine » sont introduits en France par Julia Kristeva et Tzvetan Todorov. La traduction de la Théorie esthétique d'Adorno date de 1974.

(43) Jonathan Littell, Les Bienveillantes, Paris, Gallimard, 2006, 903 p., p. 11.

(44) Claude Gauvreau, Euvres créatrices complètes, [Montréal], Éditions Parti pris, 1977, p. 228. Pour une lecture sociocritique de ce poème, voir Pierre Popovic, «Retours d'Amérique », dans Études françaises, vol. 27, $\mathrm{n}^{\circ} 1$ (1991), pp. 87-102.

(45) Claude Duchet et Isabelle Tournier, "Sociocritique », dans Béatrice Didier (dir.), Dictionnaire universel des littératures, Paris, PUF, 1994, p. 3572.

(46) Je note dans les essais de Claude Duchet que l'on trouve parfois « cotexte» au singulier parfois «cotextes » au pluriel; le singulier désigne simplement l'ensemble des liens cotextuels.

(47) Ces indices sont des clichés, des stéréotypes, des idéologèmes. 
(c'est-à-dire dotée d'une polysémie potentielle au gré du développement du texte) et systémique (le système sémiotique de l'œuvre considérée active cette charge et la modèle). Le triptyque « information, indice, valeur » aligne pour Duchet les trois « paliers méthodologiques » de sa démarche. L'activité sociogrammatique est donc un processus global de sémiotisation qui embrasse ce schéma : Réel [hors d'atteinte] / Rumeur sociale $\rightarrow$ Cotexte $\rightarrow \leftarrow$ Texte, où le cotexte médiatise le rapport du texte au référent. Au bout de la chaîne le texte agit comme un opérateur de discrétions et d'activations sémiotiques : il différencie ce qui au départ est informe, explore les possibilités de sens et en propose des nouvelles au gré de son projet scriptural, produisant de l'idéologique par l'action même de la textualité. Cette façon de procéder respecte au mieux le continuum sémiotique unissant le texte littéraire - qui, dans la pratique, est l'objet premier et dernier entrepris par la lecture - et la semiosis sociale (ici prise partiellement, à partir du texte et des liens cotextuels qu'il signale). Dans le sociogramme figure un noyau oxymorique autour duquel gravitent des oppositions multiples. Cet oxymore nodal n'est pas celui qui se déduirait d'un dictionnaire. Par exemple, "pauvre, mais honnête » est au cœur du sociogramme du pauvre sur le long XIX $\mathrm{e}^{\mathrm{e}}$ siècle (et non " pauvre, mais riche»). Les Misérables donnent un exemple de ce qu'un roman peut en faire. Écartant les bords de la plaie, Hugo pousse l'oxymoron à sa limite : " pauvre, mais honnête " devient avec Valjean "forçat, mais juste », opposition qui est une «valeur» majeure dans et pour le roman. Et l'ensemble de ce dernier multiplie à n'en pas finir les représentations contrastées (fille-mère morale, prostituée sublime, bourgeois voleur, bandit moral, etc. ${ }^{(48)}$ ) jusqu'à profiler le progressisme moral du texte sur l'écran d'une véritable encyclopédie de « la question sociale».

Analysant le roman zolien, Duchet montre qu'il lit de façon pathétique l'oxymore ville/cité, insérant entre ces deux éléments le symbole exemplaire de «Paris » et associant le premier à un mouvement et à une spatialisation où pourrait se lire l'avenir et le second à une tendance destructrice pathogène, qui ne cesse à la fois de miner et de féconder, de façon très ambivalente, la vision de l'urbanité future ${ }^{(49)}$. Dans un autre essai majeur, consacré à $L$ 'Espoir, il fait voir que l'opposition cardinale entre la Révolution et la guerre est déplacée chez Malraux par une métaphore du «bélier» connectée à un cotexte comprenant des discours révolutionnaires, un intertexte hugolien et de nombreuses connotations épiques et poétiques ; le travail romanesque de réécriture fournit la base d'une vision idéologique singulière de la guerre d'Espagne qui a elle-même une valeur historique ${ }^{(50)}$. L'approche sociogrammatique peut aussi se donner pour objet un ensemble de textes dont la publication s'échelonne sur une moyenne durée. De cette manière elle parvient à reconstruire une constellation sociogrammatique et à mettre en évidence son caractère « instable», c'est-à-dire son évolution et ses transformations continuelles. Étude systématique et très complète du sociogramme de l'écrivain, l'essai consacré par Roseline Tremblay au personnage fictif de l'écrivain dans le roman québécois des années 19601995 en est un exemple. Les projets romanesques déploient un noyau opposant «1'écrivain-écrivain » au «porte-parole » et, parallèlement, le poète à l'intellectuel. Ils en relient les éléments à plusieurs types de créateurs possibles (le « perdant », l' " aventurier », le «névrosé », etc.) et les inscrivent dans des histoires qui tendent progressivement à privilégier l'individualité plutôt que l'engagement dans les luttes de la cité(51).

(48) Ces quatre exemples renvoient à Fantine, Éponine, Thénardier, Cravatte.

(49) Claude Duchet, "Pathologie de la ville zolienne », dans Stéphane Michaud (dir.), Du visible à l'invisible. Pour Max Milner. Tome 1, Paris, José Corti, 1988, 347 p., pp. 83-95.

(50) Claude Duchet, «La manœuvre du bélier. Texte, intertexte et idéologies dans L'Espoir », dans Revue des sciences humaines, Tome LXXX, n² 204 (octobre-décembre 1986), pp. 107-131.

(51) Roseline Tremblay, L'écrivain imaginaire: essai sur le roman québécois, 1960-1995, Montréal, Hurtubise $\mathrm{HMH}, 2004,600$ p. 
La sociocritique est aussi redevable à Claude Duchet d'avoir incité ses chercheurs à travailler en microlecture. Les incipit romanesques, définis de façon restreinte comme les premières phrases des romans et de façon large comme une séquence plus ou moins longue établissant les principales conditions de lisibilité interne de ce qui va suivre, sont l'un des objets proposés à cette critique de détail. Ouverture du texte, passage " où s'échangent monde et parole, vivre et dire, nécessité et "liberté", où le choix se décide, conjointement, d'un ailleurs et d'un ici, et le profil d'un sens, dans le suspens des aut tres ${ }^{(52)}$ », entre-deux orienté « vers la réserve des possibles ${ }^{(53)}$ » autant que vers l'élan donné par les premiers mots et leur ton, « seuil à double sens, tourné à la fois vers la pa-

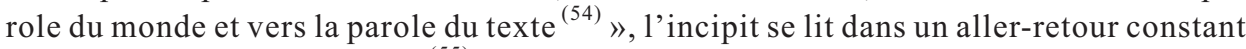
de « l'ensemble au fragment ${ }^{(55)} »$. Passible d'analyses semblables mais qui tiendront compte de toutes les ambiguïtés liées à la décision de clôturer des contes et/ou de les garder ouverts, l'explicit (excipit et desinit se rencontrent aussi) exige lui aussi une microlecture inspirée ${ }^{(56)}$. Mais se trouvent également dans le travail de Duchet des analyses serrées de cristallisations métaphoriques ou métonymiques - le bouquet d'Emma ou le motif des mains dans Madame Bovary - où sont venus se tresser des fils de la sémantique du texte.

Puisqu'elle tient que l'écriture est le lieu de la socialité du texte, la sociocritique croise volontiers la critique génétique, pour donner lieu à une sociogénétique dont Stéphane Vachon, Jacques Neefs, Pierre Laforgue ou Christian Castella ont donné de convaincantes applications ${ }^{(57)}$. Dans son examen des manuscrits, cette sociogénétique ne se borne pas, on s'en doute, à collationner les variantes, mais elle observe dans les hésitations et les ratures, dans les bifurcations et les transformations successives les traces d'une relation mouvante avec le cotexte, d'une représentation fantasmée du marché littéraire, d'une signature sertie dans les décisions esthétiques, d'une activité sociogrammatique perceptible dans les équations multiples établies entre les valeurs, les mots et les tropes. Elle « doit faire apparaître, écrit Stéphane Vachon, et les suivre pour les interroger, les marques de la socialité, les traces d'un inconscient ou les indices d'un impensé politique, les signes d'une mémoire culturelle, repérables sous les ratures, les repentirs, les refoulés, les censures, dans les virtualités et les possibles de ce qui surgit mais ne demeure pas, de ce qui s'écrit mais ne se publie pas, dans les fantômes que l'œuvre élimine pour se construire ${ }^{(58)}$.»

Les travaux d'Edmond Cros montrent que l'écriture littéraire procède à un remodelage des discours collectifs environnant le texte. Définie comme un système modélisant secondaire, la littérature exerce cette action par le moyen de traits scripturaux distinctifs

(52) Claude Duchet, "Pour une socio-critique ou variations sur un incipit », Littérature, "Littérature, idéologies, société ", $\mathrm{n}^{\circ} 1$ (février 1971), pp. 5-12, p. 9.

(53) Claude Duchet, ibid.

(54) Andrea Del Lungo, L'incipit romanesque. Texte traduit de l'italien, revu et remanié par l'auteur, $\mathrm{Pa}$ ris, Seuil, 2003, 376 p., p. 14.

(55) Claude Duchet, «Pour une socio-critique ou variations sur un incipit», loc. cit., p. 10.

(56) Claude Duchet, «Fins, finition, finalité, finitude», dans Claude Duchet et Isabelle Tournier, Genèses des fins. De Balzac à Beckett, de Michelet à Ponge, Paris, Presses Universitaires de Vincennes, 1996, 231 p., pp.6-25

(57) Claude Duchet, «Sociocritique et génétique : entretien avec Anne Herschberg Pierrot et Jacques Neefs », dans Genesis, n 6 (1994), pp. 117-127; Stéphane Vachon, "Perspectives en génétique balzacienne », dans Kazuhiro Matsuzawa (dir.), Balzac, Flaubert. La genèse de l'cuvre et la question de l'interprétation, Nagoya, Nagoya University, 2009, pp. 35-45; Charles Castella, Les contes et nouvelles réalistes de Maupassant, apôtre du marché Dieu. Lecture sociogénétique. Préface de Claude Duchet, Paris, L'Âge d'homme, 2000, 142 p. ; Pierre Laforgue, Balzac dans le texte. Études de génétique et de sociocritique, Saint-Cyr-sur-Loire, Christian Pirot, 2006, 194 p.

(58) Stéphane Vachon, "Perspectives en génétique balzacienne », dans Kazuhiro Matsuzawa (dir.), Balzac, Flaubert. La genèse de l'œuvre et la question de l'interprétation, Nagoya, Nagoya University, 2009 , pp. 35-45, p. 41. 
et par son déplacement vers des mondes fictifs. Elle déconstruit les contraintes liées à la production des discours, déstabilise le préconstruit idéologique, détourne et complexifie les syntagmes figés. Par exemple, observant la transformation de l'expression " pierres précieuses » circulant dans l'espace discursif de l'économie en l'expression " pierres de prix » dans Guzman de Alfarache de Mateo Aleman, Cros remarque que le texte assume ainsi une fonction de dévoilement : il fait en effet voir que le règne de la valeur d'échange est ordinairement dissimulé sous un voile de connotations positives et par des ressorts métaphoriques liés au mot « précieux ». Partant de là, le sociocriticien étend la lecture et découvre que toute une série de choix paradigmatiques opérés dans l'ordre des signifiants fonctionnent de la même manière et procèdent à une mise à nu critique du monde marchand et de ses rouages. En texte, la critique du discours collectif «économique » ne cesse de mettre à jour ses procédures d'embellissement, d'euphémisation et de voilement. Le « sujet», défini comme une entité repérable à l'ensemble des traces sémiotiques qui le constituent, est une notion placée au centre de la démarche critique de Cros. Les « discours collectifs » précités, qui traversent les esprits dans un moment historique donné, sont à l'actif et producteurs de « sujets transindividuels » (ils peuvent être d'ordres différents : une génération, l'ensemble des commerçants, un groupe d'intellectuels, etc.). Leur répondent des « sujets culturels », totalités subjectives issues d'un discours (d'une microsémiotique) assumé et investi par un «Je » qui se construit par et dans son interaction sémiotico-idéologique avec les « sujets transindividuels » (produits des discours collectifs). Le « sujet culturel» marque sa distance à leur égard : en raison première de ce qu'il provient de l'afflux en texte d'un inconscient psychique qui, s'il doit beaucoup à Freud, est ici articulé à un moment historique précis qui offre un lot de signifiants disponibles pour tout réinvestissement; en raison seconde de ce que le texte mêle et multiplie en son sein les temporalités historiques, convoquant des discours collectifs hétérochrones et les jouant les uns contre les autres, de sorte que se créent des béances, des discontinuités, des déphasages que la lecture a mission de mettre en évidence et d'analyser.

C'est à d'autres sources que recourt l'œuvre critique de Pierre V. Zima. Elle prend appui sur la philosophie du langage de Mikhäil Bakhtine, laquelle se fonde sur l'idée que tout énoncé est toujours déjà gros d'altérité, car les mots viennent à la parole marqués par l'usage que d'autres en ont eu, et par suite lestés d'une charge idéologique et circonstancielle. Toute parole, tout discours, tout texte reprend, répond, reconfigure du déjà-formulé/ dit/ écrit. Zima traduit cette proposition baktinienne en termes sociosémiotiques et la fait servir à l'étude de la littérature. Tout écrivain est confronté à une situation sociolinguistique, c'est-à-dire à un conglomérat de langages collectifs historiquement pris dans une interaction dynamique. Ces langages, auxquels Zima donne le nom de « sociolectes ", permettent à chaque groupe de se définir, de se projeter dans une durée (d'aucuns pointent vers le passé, d'autres vers l'avenir) et de critiquer explicitement ou implicitement les autres groupes. Un sociolecte (ce peut être le sociolecte humaniste chrétien dans les années 1950, le sociolecte mondain à la Belle Époque, le sociolecte révolutionnaire, le sociolecte psychanalytique en tel ou tel moment de l'histoire, etc.) peut ainsi « être défini comme un langage collectif marqué par un lexique, une sémantique et un faire taxinomique particuliers qui peuvent engendrer des parcours narratifs ou discours plus ou moins cohérents ${ }^{(59)}$.» Tout texte littéraire d'envergure s'offre comme une réponse active à une situation sociolinguistique historique : il « absorbe et transforme » des sociolectes hétérogènes appartenant à cette situation, les met en dialogue et en tension, les passe à la question et à la critique par des moyens précis portant sur une partie ou la globalité de leur palette langagière (sémique, phonétique, lexicale, syntaxique, sémantique, sémiotique). La seconde source majeure de l'œuvre de Pierre Zima loge à l'enseigne de l'école de

(59) Pierre V. Zima, La négation esthétique. Le sujet, le beau et le sublime de Mallarmé et Valéry à Adorno et Lyotard, Paris, Budapest, Turin, L'Harmattan, 2002, 268 p. 
Francfort, dont il a maintes fois dit qu'il respectait et adaptait le programme général : évaluer les textes littéraires devant l'histoire dans l'esprit d'une théorie critique attentive à la sédimentation du sens orchestrée par leur mise en forme. C'est envers Theodor W. Adorno que va sa plus forte dette. L'un des leviers majeurs que Zima reprend et repense à sa guise est le rabattement sur le langage de la théorie marxienne de la valeur auquel procéda Adorno dans plusieurs de ses essais sur l'art et la littérature. En régime de modernité, les mots, les signes sont en perte de valeur d'usage ${ }^{(60)}$. À l'heure du marché global, de la consommation, de la reproduction endémique des œuvres transformées en produits manufacturés, à l'heure du slogan et de la novlangue publicitaire, les mots et les signes sont eux-mêmes soumis à la valeur d'échange. Dans ses études sur le roman moderne et contemporain, Zima observe plusieurs situations sociolinguistiques successives auxquelles des œuvres romanesques marquantes offrent des réponses esthétiques fortes. Face à une situation où les signes sont employés de façon équivoque sans que personne ne s'en alarme, les romans de Kafka, Musil et Proust dévoilent les conséquences des ambivalences en installant une distance lucide dans la narration. À la recherche du temps per$d u$, par exemple, absorbe un sociolecte mondain dominé par la pratique de la conversation de salon, chérie et par les aristocrates et par les bourgeois. Le roman proustien en montre aussi bien la vanité que les conséquences froidement ravageuses et lui oppose l'authenticité de l'écriture littéraire et sa richesse d'invention, dévalorisant du même coup des esthétiques romanesques antérieures, réalistes notamment, où les dialogues rapportés tenaient un grand rôle ${ }^{(61)}$. C'est à une situation sociolinguistique saturée de mots-valeurs désémantisés par les jeux politiques que sont affrontés des écrivains comme Sartre, Moravia et Camus. Ces romanciers incorporent dans leurs œuvres des sociolectes politico-idéologiques dévitalisés et, avec des moyens différents, en examinent sans concession les effets dévastateurs. Dans L'Étranger, l'écriture de Camus annexe le sociolecte humaniste-chrétien dont se soutient le discours de la justice. Il le donne à entendre avec ironie et montre son incapacité à accepter l'idée que Meursault, le héros du roman, puisse simplement être indifférent à ses charmes rhétoriques et conscient de son caractère arbitraire et contingent ${ }^{(62)}$. En régime de postmodernité, la situation sociolinguistique se caractérise par la surabondance et par une interchangeabilité générale des signes, des valeurs et des énoncés. Quatre types d'esthétique romanesque répondent à cet état des langages sociaux. Comme s'ils s'efforçaient d'éviter le pire, des textes, à l'exemple de ceux d'Ernest Callenbach, intègrent des sociolectes idéologiques (l'écologie, le féminisme) mais en les débarrassant vigoureusement de leur caractère épiphanique ou millénariste. Robbe-Grillet et les nouveaux romanciers recyclent des sociolectes d'avant-garde et développent des fictions narratives expérimentales qui ne cessent d'explorer les conséquences d'un abandon de toute recherche métaphysique ou utopique. Entée sur les discours d'érudition et renonçant au sérieux de la tradition romanesque, une troisième voie dont l'œuvre d'Umberto Eco est exemplaire propose des romans ouverts aux savoirs et au ludisme. Enfin, se nourrissant, parfois jusqu'à la désolation la plus amère, d'une manière de sociolecte postmoderne global (renoncement à tout récit d'émancipation collective, individualisme, relativisme, « absence de repères »), des romans tels ceux de Werner Schwab, Christoph Ransmayr ou Thomas Pynchon en effectuent une critique radicale, pointant une autodestruction du monde intériorisée par des individus

(60) Ils n'ont plus de valeur en eux-mêmes, comme ils pouvaient en avoir dans un autrefois quelque peu idéalisé, ce dont témoigne leur aura sémantique dans la grande poésie qui accompagna 1'Aufklärung par exemple.

(618) Pierre V. Zima, L'ambivalence romanesque : Proust, Kafka, Musil. Nouvelle édition revue et augmentée, Paris, L'Harmattan, 2002, 393 p.

(62) Pierre V. Zima, L’indifférence romanesque : Sartre, Moravia, Camus. Deuxième édition revue et corrigée par l'auteur, Montpellier, Éditions du C.E.R.S., 1988, 232 p. 
qui ne semblent pas avoir de prise critique à son endroit ${ }^{(63)}$. Ce résumé trop rapide de l'œuvre de Pierre V. Zima donne, du moins je l'espère, un aperçu de son ampleur de vue.

Omniprésent autrefois, le concept d'idéologie a quasiment disparu de la carte dans le domaine des sciences humaines ${ }^{(64)}$. Pierre V. Zima et nombre de sociocriticiens l'ont également abandonné, préférant manier des concepts tels ceux de langage ou de discours. Plusieurs critiques ont cependant tenté de renouveler l'analyse idéologique en abandonnant le préjugé de « fausse conscience ». Le principal d'entre eux est Philippe Hamon. Dans une démarche qui s'adresse au « dedans du texte », Hamon analyse " l'effet-idéologie », lequel résulte de la mise en place d' « appareils normatifs-évaluatifs » dans un texte. Cette normativité et cette évaluation sont repérables et distribuées dans les multiples aspects des modes énonciatifs : elles peuvent être à l'actif du narrateur, être implicites ou déléguées à des personnages, relever de notations disséminées ou de non-dits orchestrés dans le flux de la prose, etc. Leurs manifestations entretiennent des relations complexes dont il s'agit de rebâtir le système pour prendre mesure de l'effet-idéologie. Ce « discours d'escorte évaluatif» dont sont nattés les rapports entre les actants est le premier objet d'une lecture qui est constamment à l'affût de toutes les traces de jugement. Quand le narrateur des Misérables qualifie les lectures de Madame Thénardier en ces termes : «Or on ne lit pas impunément des niaiseries », quand Gavroche gratifie Valjean d'un adjectif qui montre sa vaste connaissance de l'humanité : "Vous êtes un brave homme », ce sont autant de marques à recenser. Hamon montre bien que ces traces sont très variées et peuvent toucher la langue, le goût, l'esthétique, le politique, l'éthique, etc. Nul doute qu'une telle approche ferait son miel de nombre de romans contemporains, à commencer par ceux de Michel Houellebecq, où les « appareils normatifs-évaluatifs » sont surdimensionnés et littéralement prosophages. Elle relève de la sociocritique dès lors que cet effet-idéologie est rapporté à des " univers de valeurs » incorporés dans les discours produits par la société sociale environnante ${ }^{(65)}$. Une partie du travail récent de Marc Angenot, placée en ce point où se rencontrent théorie de l'argumentation, analyse du discours et histoire des idées, propose elle aussi un renouveau de l'analyse idéologique. Prenant à bras le corps des thèmes ou dispositifs idéologiques complets, ce travail montre que leur cohérence est largement de parade ou de convention et qu'ils comprennent des amphibologies, des paralogismes, des apories du raisonnement qui inscrivent dans leur matière les limites temporelles de leur séduction ${ }^{(66)}$. Ce travail d'analyse intéresse au plus haut point une sociocritique désireuse de montrer comment l'art contredit l'idéologique ou le déstabilise assez pour entamer son crédit potentiel.

Lieu dynamique de l'essor de la sociocritique, L'École de Montréal se caractérise par le soutien que ses initiateurs trouvèrent dans les travaux de Mikhaïl Bakhtine. Faisant fond sur le postulat d' « une interaction générale des énoncés », ils mirent à profit tous les éléments fondamentaux de la critique bakhtinienne : l'examen des relations entre culture populaire et littérature (le carnavalesque et le grand essai sur Rabelais), l'étude du dialogisme, la mise en évidence des chronotopes, la prise en compte de tout ce qui dans la matière textuelle relève de l'hétérogène et du multiple (polyphonie, plurilinguisme, polysémie, etc.), et surtout l'apport déterminant des concepts d'intertexte et d'interdiscours. Ces derniers permettaient de substituer à des conceptions arrêtées des textes chérissant

(63) Pierre V. Zima, Théorie critique du discours. La discursivité entre Adorno et le postmodernisme, Paris, Budapest, Turin, L'Harmattan, 2003, 187 p.

(64) Ce qui, on l'aura peut-être remarqué, n'est pas le cas de l'adjectif "idéologique».

(65) Philippe Hamon, Texte et idéologie. Valeurs, hiérarchies et évaluations dans l'œuvre littéraire, Paris, PUF, 1984, $227 \mathrm{p}$.

(66) Voir entre autres Les idéologies du ressentiment (Montréal, XYZ Éditeur, 1995), Les grands récits militants des XIX et XX $X^{e}$ siècles. Religions de l'humanité et sciences de l'histoire (Paris, L'Harmattan, 2000), La démocratie, c'est le mal. Un siècle d'argumentation anti-démocratique à l'extrême gauche (Québec, Presses de 1'Université Laval, 1994), etc. 
leur linéarité et leur « autonomie » une pensée active privilégiant la transformation et l'action : les romans de Rabelais ingèrent tous les discours du savoir de leur temps, non pour en tresser une courtepointe ou faire preuve d'érudition stérile, mais pour en faire autre chose en laissant donner en langue tous les moyens sémiotiques et rhétoriques issus des sources populaires du carnaval ; les œuvres de Dickens ou de Dostoïevsky captent mille et une voix parlant de la misère ou de l'argent, non pour en faire simple collection, mais pour les redonner à entendre autrement par le moyen du dialogisme. Les essais d'André Belleau et de Gilles Marcotte sur le roman et la poésie sont des illustrations remarquables de cette écoute de la diversité interne des œuvres littéraires ${ }^{(67)}$.

À leur côté ou dans leur foulée, dans un prolongement novateur des ouvertures de Bakhtine, les débats engagés par l'articulation de la sociocritique et de la théorie du discours social de Marc Angenot ont servi de voile de misaine à nombre de sociocriticiens.

1889. Un état du discours social est la principale pièce de charpente d'un imposant ensemble d'articles et d'essais ${ }^{(68)}$ issus d'une recherche de longue haleine portant sur l'état du discours public à la fin du XIX ${ }^{\mathrm{e}}$ siècle dans l'espace géoculturel de la francophonie européenne (France, Belgique, Suisse romande). Effectuant une coupe synchronique sur l'année $1889^{(69)}$, Angenot prend en compte la totalité de la chose imprimée produite dans l'espace public ${ }^{(70)}$. Ce « discours social» est d'abord l'objet d'une définition empirique, puisqu'il désigne « tout ce qui se dit et s'écrit dans un état de société donné ». A priori rendre raison de tout l'éventail du dicible et de l'inextricable fouillis que chacun imagine paraît de l'ordre de l'impensable. À contre-courant de cette impression, Angenot démontre que c'est une illusion entretenue par le discours social lui-même que de laisser croire que tout peut se dire et que la différenciation des choses dites serait infinie. Pour lui, l'une des fonctions de la socialisation du discursif est d'imposer des contraintes et des règles délimitant ce qui peut être publiquement dit ou non, ainsi que des façons tenues pour acceptables de débattre et de raconter. Le postulat bakhtinien d'une "interaction généralisée » des énoncés est incliné vers une perception qui privilégie la circulation, l'itération, l'essaimage des mots et des représentations. L'analyse de l'interdiscursivité générale tend à montrer que, loin d'être d'une variété infinie, le discours social est soumis à un nombre restreint de «mécanismes unificateurs et régulateurs » en sorte qu'il se présente comme une "hégémonie », laquelle consiste en un « ensemble de règles prescriptives de diversification [...] et de cohésion, de coalescence, d'intégration [des choses dicibles] ${ }^{(71)}$. » Pour le mettre en évidence, le chercheur abolit les hiérarchies symboliques habituelles : au moment de l'analyse, les textes de littérature, le récit d'actualité, la presse boulevardière, l'essai scientifique, le tract électoral sont mis sur le même plan, examinés du même point de vue panoramique et confrontés les uns aux autres sans préjugement de classement. Cette mise à plat donne à comprendre qu'ils sont pris dans des relations de contiguïté et de similarité et que, si par aventure un texte ou un discours est original, sa particularité ne se gagne et ne se mesure que par rapport à l'hégémonie globale : en d'autres termes, celle-ci produit la platitude, le commun et le trivial mais aussi, par rétroaction, le singulier et l'original. La description du discours social de 1889 se déploie en conformité avec ces principes méthodologiques. L'hégémonie est décomposable en sept traverses

(67) André Belleau, Le romancier fictif, Montréal, PUQ, 1980, 155 p. ; Gilles Marcotte, Le roman à l'imparfait (Montréal, La Presse, 1976, 194 p.), Littérature et circonstances (Montréal, L’Hexagone, 1989, 350 p.), La prose de Rimbaud (Montréal, Boréal, 1989, 193 p.).

(68) Ce que l'on dit des Juifs en 1889 : antisémitisme et discours social, Le cru et le faisandé : sexe, discours social et littérature à la Belle Époque, Le centenaire de la Révolution, Topographie du socialisme français.

(69) Année innervée par plusieurs événements marquants (centenaire de 1789, inauguration de la Tour Eiffel et Exposition universelle de Paris, crise politique du boulangisme).

(70) Si la saisie angenotienne est déjà très large, elle l'avait été plus encore dans le Tome 6 de la somme de Robert Fossaert sur La société : cf. Les structures idéologiques, Paris, Seuil, 1983, 620 p.

(71) Marc Angenot, 1889. Un état du discours social, Longueuil, Le Préambule, 1989, 1167 p. 
contraignantes. 1. La première contrainte est l'imposition des formes acceptables de la langue : la langue littéraire - qui excède la littérature - est tenue pour l'étalon et la quintessence de la « langue nationale». 2. L'hégémonie discursive de 1889 comporte une topique formée d'une concaténation de lieux communs et de présupposés qui régentent les prises de parole et se retrouvent partout, et qui dérivent d'un noyau thématique faisant voir le monde et la société sous l'angle d'une décadence en cours. À ce noyau thématique, Angenot donne le nom de «paradigme de la déterritorialisation » : véritable «métastase », il se décline sous de multiples formes dans les différents secteurs discursifs. Il se dit ainsi ad libitum que le progrès va tout broyer, que la langue française dépérit, que le «moi » se décompose, que les esprits sont détraqués, que la ville est une démone, que l'économie périclite de crise en crise, que l'art n'est plus ce qu'il était, que le sexe féminin disparaît (certaines femmes portent des pantalons ou fument), que des périls (jaune, vénérien) sont «à nos portes ». Le discours social de 1889 exploite à n'en pas finir le motif de la fin (d'une race, d'un monde, d'un sexe, de la France, du sens, du vrai, de l'authentique, du stable, de la cuisine saine $)^{(72)}$. 3. La topique de la décadence est soutenue par une "gnoséologie romanesque ", ce qui revient à dire que la manière commune de connaître le monde nécessite de la mise en récit. Dans cette logique, le roman littéraire est la conséquence de cette tendance gnoséologique. 4. L'hégémonie fin de siècle, ce sont aussi des «fétiches », dont il est interdit de dire du mal, comme la patrie, le drapeau national, l'armée, la science positive, ou des « tabous », objets de délectation morose et de fascination hypocrite dont tout le monde parle pour dire qu'il est impensable d'en parler, telles la folie, l'hystérie, les perversions sexuelles. 5. La « chose imprimée » n'est accessible qu'à un énonciateur légitime, lequel est « Français, adulte, mâle, lettré, urbanisé, en pleine entente complice avec le jeu des thématiques dominantes. " Par contrecoup, elle comporte des mécanismes d'exclusion suractifs : là se rencontrent l'égocentrisme, l'ethnocentrisme, les classocentrismes, le sexisme de la doxa de 1889. Le discours social fin de siècle tient par certains côtés d'une entreprise xénophobe et chauvine, porteuse d'un antisémitisme très présent et d'une germanophobie issue de la guerre de 1870. 6. Entée sur l'imaginaire de la décadence, la «dominante de pathos » du discours fin de siècle est l'angoisse. 7. À ces six composantes s'en ajoute une septième qui s'oppose aux autres de la même façon que le pouce s'oppose aux autres doigts de la main. Chaque secteur du discours use de la topique et des mécanismes de régulation susmentionnés selon ses intérêts, selon ceux auxquels il s'adresse, selon sa tradition et son idéologie propres. Cette « division du travail discursif » fait voir que le même motif, la prostitution par exemple, se rencontre sous des allures particulières dans la presse boulevardière (qui parlera des « cocottes » et des «horizontales » en usant du sous-entendu comme moyen rhétorique), la science (qui en fera des études statistiques dont furent friands les hygiénistes) ou la poésie (qui en fait une allégorie fascinante). Dans ce modèle théorique ambitieux, mettant en coupe la totalité de la sémiosis sociale, c'est la socialisation interactionnelle du discours qui fait qu'il répond à un certain nombre de contraintes et de règles délimitant ce qui peut être publiquement dit ou non, de même qu'elle impose des façons tenues pour légitimes de débattre et de raconter. L'une des critiques majeures faites à ce travail est qu'il privilégie les reconductions du même au détriment du repérage du neuf, du contradictoire, du dissident ou, plus modestement, du particulier. La préséance accordée en cours d'analyse à la thématique et à la théorie de l'argumentation ${ }^{(73)}$ aggrave un nivellement des énon-

(72) Il y en a pour tous les goûts : «tout le monde peut se situer en un point de la déterritorialisation sans en assumer toute la logique redondante », ce qui signifie que le "péril vénérien » et le "péril jaune ", même s'ils apparaissent à des endroits séparés sur la carte du discours, participent néanmoins d'une même production de croyance et que l'un, d'une certaine façon, favorise la peur associée à l'autre.

(73) Des fictions peuvent ainsi être ramenées à un schéma argumentatif déduit d'un simple alignement de thèmes, leur écriture apparaissant dès lors comme un ornement sémiotiquement inerte. 
cés ${ }^{(74)}$ par rapport auquel la sociocritique, toujours à la recherche de la singularité potentielle des textes (elle est bien une " critique ", c'est-à-dire une évaluation), niche dans une contradiction dont le dépassement peut prendre deux voies.

La première est indiquée par Angenot lui-même. Même si ses commentaires sont fréquemment expéditifs quand il parle littérature, il valorise néanmoins trois types de textes. Il célèbre des inventeurs de langage, dont Proust, Kafka et Musil, reconnaît que la littérature peut introduire un «écart productif » au milieu de la mêlée des énoncés sous la forme d'un travail de brouillage, d'opacification, de détournement des évidences et des argumentaires, tient que, dans sa meilleure veine, la littérature sait que la vérité n'est pas de ce monde. Elle peut alors user d'une négativité latérale opposant aux croyances et aux errances du sens commun le fait que les façons dont le monde est connu sont le plus souvent illusoires et éphémères.

La deuxième voie convie à inverser les moteurs et à tenir le pari herméneutique qui est au centre de la sociocritique. Actant que la textualisation se distingue de la discursivité, il s'agit d'aller du texte au discours social, et non l'inverse, pour la bonne raison que c'est là le meilleur moyen de ne pas surévaluer le même et de ne pas sombrer dans l'illusion du redondant. Si on la lit vraiment, une «nouvelle en trois lignes » de Félix Fénéon n'est pas un simple « fait divers » commun. Un texte littéraire digne de ce nom - il revient à la lecture de le montrer - déplace le préconstruit, déstabilise le doxique, contredit les règles de l'acceptable, excède les permissions ou les interdits de l'opinable. L'œuvre de Régine Robin est exemplaire de cette démonstration. En son point de départ, un test-repoussoir : Le réalisme socialiste. Une esthétique impossible ${ }^{(75)}$. Mettant en coupe le corpus romanesque publié en russe durant les années trente alors que s'impose la dictature stalinienne, cet essai montre que les choix esthétiques des romans souscrivant au réalisme socialiste respectent un monologisme culturel exigé par leur inféodation à une thèse idéologique ayant valeur de vérité transcendante (thèse épousant très vite « la ligne du Parti »). Ces romans sont par suite privés des libertés de l'imagination littéraire ; ils relèvent d'une esthétique impossible, puisqu'elle vise à exclure toute hybridation formelle, tout écart, tout doute, tout suspens ou toute dérive du sens. La démarche intellectuelle subséquente de Robin la mène à des écritures cultivant ces libertés. Elle les trouve dans des prises de distance efficaces à l'égard de tout ce qui relève du propre, de l'un, du pur, de l'homogène. $K a f k a^{(76)}$ définit l'image de textes naviguant en permanence dans un entredeux, s'ouvrant vaille que vaille un espace d'indécidabilité à coups de dérives polysémiques et de suspensions réitérées du sens. Les trois essais qui suivent composent une forte trilogie. Le deuil de l'origine ${ }^{(77)}$ part de l'idée que l'écrivain est par excellence celui qui est en rupture avec la foi en l'existence d'une langue maternelle fixe et autarcique. Les textes de Nabokov, Green, Beckett, Freud, Canetti, Kerouac sont toujours aux prises avec des pluralités et des altérités langagières mais, de ce fait, ils inscrivent en prose la condition de l'individu social moderne et contemporain. Le même esprit habite Le roman mémoriel $^{(78)}$ et Le Golem de l'écriture ${ }^{(79)}$, lesquels enregistrent l'entrechoc des mémoires individuelles et collectives et l'émergence de nouvelles formes de l'invention de soi (autocréations pseudonymiques, simulations et virtualisations, créations cybernétiques, récits autofictionnels, etc.). Pour l'auteure de La mémoire saturée ${ }^{(80)}$, la littérature est en prise directe sur le vaste carnaval de représentations que les individus se montent pour

(74) Pour une critique à la fois empathique et sérieuse de ces travaux, voir Yan Hamel et Emmanuelle Jacques (dir.), «1889 a eu vingt ans. Questions à Marc Angenot», Discours social, vol. 36 (2010), 51 p.

(75) Paris, Payot, 1986, 347 p.

(76) Paris, Belfond, 1989, 347 p.

(77) Paris, Presses universitaires de Vincennes, 1993, $270 \mathrm{p}$.

(78) Le roman mémoriel : de l'histoire à l'écriture du hors-lieu, Longueuil, Le Préambule, 1989, 196 p.

(79) Le Golem de l'écriture : de l'autofiction au cybersoi, Montréal, XYZ, 2005, 288 p.

(80) La mémoire saturée, Paris, Stock, 2003, 524 p. 
essayer de dire quelque chose du réel qui ne serait pas du délire. Les travaux récents de Régine Robin ont continué à suivre les subjectivités composites, positivement déjantées, apparaissant dans le monde actuel et activées par des écritures littéraires trouvant dans les mixtions de genres et de corpus, ainsi que dans des rencontres choisies avec certaines technologies de pointe, des ressources inusitées d'invention. Ils se sont aussi lancés dans deux directions. D'une part vers une lecture du devenir actuel du flâneur baudelairien, que Berlin Chantiers ${ }^{(81)}$ annonçait, et qui a débouché sur Mégapolis, un essai où le chaos, l'entropie, la «déglingue » des mégapoles, loin d'être tenus pour dommageables, sont vus comme des lieux de déambulation infinie, comme des démultiplicateurs de possibilités et d'opportunités, susceptibles de donner naissance à de nouveaux cadres interprétatifs de la vie individuelle et de la vie sociale ${ }^{(82)}$. D'autre part vers une analyse de la production de l'oubli dans les sociétés modernes : comment se passe le refoulement des passés délicats ? quelles logiques président à l'effacement sélectif des traces ? quelles soustractions accompagnent les procédures d'archivation? En tout ce travail, que domine un refus radical du biographique et du déterminisme ${ }^{(83)}$, la lecture repose sur trois choses : un principe d'immersion (il faut squatter les textes, les habiter et comprendre leur logique de l'intérieur, penser un temps avec leurs expressions), un principe de nomadisme cognitif (adjonction de savoirs divers selon la nécessité, du moment que ces recours servent la visée herméneutique choisie), un principe de concrétude (l'intersémioticité du texte ne tourne pas sur elle-même ; collé à la mêlée des mots et des images, le texte métabolise ce qu'il lui emprunte en sorte que ce qu'il dit et donne pour vrai possède un impact tangible sur la vie quotidienne et s'inscrit de mille manières dans l'expérience humaine du monde $\left.{ }^{(84)}\right)$.

L'attention portée par Régine Robin à l'activité mémorielle des œuvres littéraires offre un thème de recherche fertile à la lecture sociocritique. Nombre de romans fédèrent un conflit de récits, ostensibles, esquissés ou latents selon les cas, corrélés dans la narration à des temps différents ou à des visions du présent, du passé et de l'avenir concurrentiel les ${ }^{(85)}$. Yan Hamel a traité cette problématique en étudiant la thématisation de la Seconde Guerre mondiale dans une vingtaine de romans publiés entre 1945 et 2000. Distinguant quatre types de représentation romanesque de la guerre - le roman résistancialiste, le roman anti-résistancialiste, le roman de la conscience inquiète et le roman de la mémoire auto-réflexive -, il montre que chacun d'eux est porteur de la proposition d'une «mémoire collective », mais qu'il est aussi tiraillé par des oppositions internes, troué de failles, lesté de dénis en raison de la complexité et des tensions afférentes au projet de raconter un événement aussi complexe et traumatique ${ }^{(86)}$.

(81) Berlin Chantiers : essai sur les passés fragiles, Paris, Stock, 2001, 446 p.

(82) Mégapolis. Les derniers pas du flâneur, Paris, Stock, 2009, 397 p.

(83) Car «l'essentiel n'est pas de chercher des déterminations “dans la vie" qui seraient à l'origine de l'œuvre et que le texte retraduirait, transposerait dans l'univers des signes du langage » (Kafka, p. 18).

(84) Dans ses essais sur la littérature urbaine, Robin double la lecture in vivo des textes de sa propre connaissance des villes, née de ses déambulations dans les bars, les musées, les cinémas, les parcs et les rues. Au texte sur la ville se lie la lecture de la ville-texte. Cet appui sur l'expérience personnelle des choses justifie l'intégration de la création littéraire dans l'exercice de la critique. De La Québécoite (Québec/Amérique, 1983) à L'immense fatigue des pierres (XYZ, 1997) et aux récentes Cybermigrances (VLB éditeur, 2004), nombre de récits et de nouvelles sont insérés dans les ouvrages à dominante critique. Cette insertion signale que la fiction est aussi un outil de connaissance de la socialité du moi. Elle n'en est un cependant que si les choix esthétiques gouvernant la mise en texte sont compatibles avec les objectifs de la sociocriticienne, d'où, par exemple, le recours au collage, au montage, à l'assemblage, à la mixtion des temporalités dans des proses narratives portant sur le devenir de la mémoire en régime de postmodernité.

(85) Une semblable pluritemporalité s'exprime dans les œuvres théâtrales ou poétiques, avec des moyens sémiotiques différents.

(86) Yan Hamel, La bataille des mémoires. La Seconde Guerre mondiale et le roman français, Montréal, Presses de 1'Université de Montréal, 2008, 410 p. 
L'étude des liens entre le texte littéraire et un discours social, dont il est traversé, mais sur les topiques, représentations et configurations duquel il peut avoir une prise critique et exercer un travail de distanciation productif, peut gagner à se déployer dans une moyenne durée. C'est ce que je me suis efforcé de démontrer dans un essai consacré à trois recueils de poésie publiés au tournant des années 1950 par Claude Gauvreau (Étal mixte), Anne Hébert (Le tombeau des rois) et Gaston Miron (les poèmes repris dans Deux sangs, écrit avec Olivier Marchand ${ }^{(87)}$ ). L'analyse des poèmes y est projetée sur l'évolution du discours social québécois telle qu'elle s'est déroulée sur une vingtaine d'années entre le milieu des années trente et le milieu des années cinquante. Concentrée sur une série de formations discursives échelonnées sur cette période et tirées de sphères très diverses de la vie publique (textes d'idéologues, discours politique, manifeste d'avant-garde, dépliants de maisons éditoriales, articles de journaux, programme des premiers psychanalystes québécois, etc.), l'étude de cette évolution met en exergue une tendance lourde qui conduit à mettre en cause le modèle historico-épique dont était encore gros le discours social des années trente. Une nouvelle façon de donner sens à la vie de la société et à son histoire, l'installation entre le nous historique traditionnel et l'individu s'exprimant au je d'un espace d'indécision où s'aventurent, au nom de la lucidité raisonnante, de la complicité ou du désir, de petits groupes s'efforçant de conduire une action concrète sur leur ou le milieu, une manière d'ouverture à la spontanéité, à la volonté d'individuation, à l'élan intérieur, voire à quelque mysticisme politique, une acceptation du risque d'entreprendre et de penser, toute une série d'indices indiquent une mutation progressive de l'hégémonie doxique des années trente. Que disent les poésies de Gauvreau, Hébert, Miron par rapport à ce mouvement? Elles accompagnent l'aspiration au renouveau, sans aucun doute, mais d'une manière qui n'est qu'à elles et de façon doublement contradictoire. D'une part elles remobilisent le grand récit épique lors même qu'elles le mettent à mal : Gaston Miron esquisse une réconciliation des morts et des vivants scellée dans un instant de recueillement constamment reporté dans le futur, Anne Hébert inverse les signes et introduit au féminin le flambeau du désir et de l'action, Claude Gauvreau fait du poète excentré le nouveau héros d'une épopée colossale. D'autre part elles proposent pour guides des valeurs étoilées d'utopicité, très différentes de celles diffusées par les discours novateurs qu'elles accompagnent : à la raison lucide, à l'engagement fraternel du petit groupe dans le milieu, à la foi dans la création collective, elles substituent ou opposent respectivement le rêve de repossession d'un espace sensible frappé d'incomplétude (Miron), l'offrande d'un don nombrable de soi (Hébert), l'image grandiose d'un sujet pathétique que chacun pourrait devenir (Gauvreau). Et plus encore, elles cherchent et trouvent dans la langue poétique une façon d'aller chercher des signes plus anciens que le passé cadastré par l'histoire officielle, du côté des archaïsmes (Miron), de signes appartenant à la mythologie égyptienne (Hébert), d'une Amérique d'avant sa découverte (Gauvreau) ${ }^{(88)}$.

Le concept d' « imaginaire social » peut, au prix d'une redéfinition dont Jean-Charles Falardeau avait donné une première mouture dans un essai en son temps très innova teur ${ }^{(89)}$, servir la lecture sociocritique des textes ${ }^{(90)}$. J'en ai tenté l'expérience dans le but

(87) Olivier Marchand et Gaston Miron, Deux sangs, Montréal, L’Hexagone, 1953, 67 p. ; Anne Hébert, Le Tombeau des rois, Québec, Institut littéraire du Québec, 1953, 76 p. ; Claude Gauvreau, Étal mixte (1950-1951), dans Euvres créatrices complètes, [Montréal], Éditions Parti pris, 1977, pp. 211-265.

(88) La contradiction du poème. Poésie et discours social au Québec de 1948 à 1953, Candiac, Éditions Balzac, 1992, $455 \mathrm{p}$.

(89) Jean-Charles Falardeau, Imaginaire social et littérature, Montréal, Hurtubise HMH, 1974, 152 p.

(90) Cette redéfinition est à la fois très éloignée de l'usage banalisé d'expressions comme «imaginaire romanesque » ou « imaginaire de [la ruine, la mer, la ville, etc.]» ou « imaginaire collectif » commun dans la prose courante. Elle est aussi sans lien direct avec l'usage précis et cohérent qui en est fait dans la philosophie de Cornelius Castoriadis (dont l'intention de sortir du simple déterminisme matériel et historique est cependant une source féconde d'inspiration). 
de trouver un moyen de rendre justice au caractère mouvant, labile, imprévisible - hormis après-coup - de toute semiosis sociale dès lors qu' on l'observe dans son historicité. À ce but premier de construire un concept plus souple que celui de « discours social» s'est adjoint le constat que la façon dont les individus et les groupes tentent de dire ce qu'ils sont et ce qu'ils vivent marche bien davantage aux effets d'une rhétorique de la séduction (axée à la façon du Groupe Mu sur l'elocutio) qu'au produit d'une logique ou d'une topique argumentative ${ }^{(91)}$. Une tierce intention résultait d'un doute de plus en plus prononcé à l'égard de cette mauvaise habitude héritée à la fois de la religion austère d'autrefois et des courants théoriques des années soixante-dix, voulant que ce soit toujours quelque chose (le démon, l'idéologie dominante, la classe sociale, une institution, etc.) qui parle à travers quelqu'un que ce quelque chose agit et manipule : il fallait en conséquence tenter de penser le texte et la semiosis environnante de manière réellement dynamique, comme des objets réellement interactifs et productifs d'une action résultant de leur interactivité même. Enfin, le réflexe de s'en remettre à la sociologie dès qu'il est question de raccorder « le social et le littéraire » me paraissait une coutume non nécessaire. Depuis cent ans et plus, les études et la théorie littéraires recèlent d'armes et de moyens qui ne demandent qu'à servir en dehors de leurs cases disciplinaires académiques; la sociocritique, qui ne cesse de montrer que, sur le plan où elle se place, la littérature n'est nullement autonome par rapport au monde ni tel qu'il va ni tel qu'il est dit, est idéalement placée pour procéder à cette exportation.

L'imaginaire social est ce rêve éveillé que les membres d'une société font, voient, lisent et entendent et qui leur sert d'horizon de référence pour tenter d'appréhender, d'évaluer et de comprendre la réalité dans laquelle ils vivent. Il peut être pensé comme suit:

\section{Ensembles de représentations et fictions structurantes}

L'imaginaire social est composé d'ensembles interactifs de représentations corrélées, organisées en fictions latentes ${ }^{(92)}$, sans cesse recomposées par des propos, des textes, des chromos et des images, des discours ou des œuvres d'art. Par exemple, le bonapartisme et le positivisme forment deux de ces ensembles dans l'imaginaire social du second Empire, et les deux fictions qui les structurent ont plus d'un point commun. Dans toute société, quatre de ces ensembles de représentations sont essentiels : le premier concerne l'histoire et la structure de la société (représentations du passé, du présent et de l'avenir ; représentations des institutions, des hiérarchies, des collectivités); le deuxième la relation entre l'individu et le collectif global (représentation de l'individu, de sa vie, des rapports du privé et du public); le troisième la vie érotique (représentations des corps, des affects, des sentiments, du sexe); le quatrième le rapport avec la nature (représentations métaphysiques, religieuses ou non religieuses, etc.).

\section{Modes de sémiotisation: une littérarité générale}

Il est de notoriété critique qu'aucune définition endogène n'a pu définir de manière parfaitement satisfaisante ce que c'est que la littérarité d'un énoncé. Dès lors, de deux choses l'une : soit la littérarité n'existe pas, soit il n'y a que cela et elle reste indéfinissable parce qu'elle habite de part en part notre expérience langagière du monde. Choisir la seconde option conduit à voir l'imaginaire social comme le résultat de l'action de cinq

(91) D'autres dimensions seraient à ajouter comme l'interaction avec la « réalité » ou la polémicité interne du concept. Pour plus de détails, voir Pierre Popovic, Imaginaire social et folie littéraire. Le second Empire de Paulin Gagne, Montréal, PUM, 2008, 377 p.

(92) Des fictions au sens propre, avec des héros, des traîtres, des aventures, des débuts, des fins, des recommencements, etc. 
modes majeurs de sémiotisation de la réalité : 1 . une narrativité qui, d'une part, conduit à l'émergence de fictions latentes et, d'autre part, à l'édification de héros, lesquels peuvent être mythiques, transformés par «leur» légende ou tirés de la vie réelle ; 2 . une poéticité qui multiplie les figures de sens, métaphores, métonymies, synecdoques (et al.), et diffuse des siginifiants-phares, ainsi que des rythmes de mise en parole ; 3. des régimes cognitifs, c'est-à-dire des façons de connaître et de faire connaître, qu'elles soient diffusées par la presse ou par des traités académiques, qu'elles soient d'ordre mythologique ou religieux, qu'elles appartiennent ou non à ce qui est appelé « science » ou reconnu comme savoir légitime à tel ou tel moment de l'histoire ; 4. une iconicité, car l'imaginaire social, c'est aussi tout un imposant matériel d'images, de caricatures, de photos, de peintures, et aujourd'hui de films, de clips et de sites, dont l'ère moderne ou contemporaine assure la reproductibilité sur grande échelle ; 5 . une théâtralité, visible dans le cérémonial privé, politique, culturel, militaire, dans les célébrations, les rituels, les parades, les gestuelles, les scénographies sociales.

La distribution en cinq modes - narrativité, poéticité, cognitivité, icônicité, théâtralité - signifie que l'imaginaire social est empreint de littérarité. De là découle une façon particulière de reposer la question nodale de la sociocritique. Ce qui est appelé « littérature » est ce qui résulte d'une formalisation problématique de l'imaginaire social, aussi bien au niveau du système générique qu'au niveau des textes : un roman est en interaction dynamique majeure avec la narrativité ambiante ${ }^{(93)}$, c'est-à-dire avec les façons de raconter l'histoire de la société et les événements qu'elle vit. La mise en texte peut sans doute simplement calquer ces façons de raconter, mais elle vise plus souvent - dans les sociétés qui le permettent - à les détourner, à les critiquer, à les transformer. Toute mise en forme vivace est alors dite problématique parce que les textes littéraires sont susceptibles d'installer une distance sémiotique à l'intérieur et à l'égard de cet imaginaire social justement pour la raison qu'ils activent, individualisent les cinq modes de sémiotisation prédécrits ${ }^{(94)}$. Ils sont clairement dans un continuum sémiotique avec lui. Ils en dérivent, ils le travaillent, ils le pourvoient. L'objectif propre de la sociocritique consiste à mesurer cette distance sémiotique sur le fond de ce continuum et à la comprendre.

Dans cette approche, tant les fictions latentes structurant l'imaginaire social que les textes étudiés doivent être soumis à une lecture littéraire (ou sémiotique si l'on préfère). De cette manière il est loisible de montrer que L'Unitéide (1857) de Paulin Gagne, énorme épopée narrative versifiée racontant la venue de la fille de Dieu sur terre et son combat contre les démons qui précipitent l'humanité vers une mort certaine, répond d'une logique narrative parfaitement compossible avec les récits dont se soutiennent le bonapartisme, le catholicisme et le comtisme. Tous quatre enchaînent quatre étapes narratives : une faute originelle déterminante, la nécessité d'une réparation, l'arrivée du sauveur providentiel et l'annonce du salut universel. Sous Louis-Napoléon Bonaparte, la réalité sociohistorique est notamment connue au tamis de ce récit unique et pourtant multiforme. L'Unitéide de Gagne offre la particularité de gorger cette fiction de pathos et d'hystérologie, la poussant à la limite et lui inoculant un fantasme sacrificiel morbide dont une microlecture appliquée peut montrer les tenants et les aboutissants.

Tout sociocriticien lisant les travaux de Jean-Marie Privat et de Marie Scarpa se croit un peu comme M. Jourdain à l'égard de la prose et se dit qu'il a souvent fait de l'ethnocritique sans le savoir, surtout s'il observe la direction prise par ces travaux au cours des dix dernières années. Le coup d'envoi de l'ethnocritique est donné dans l'essai que publie

(93) Pas seulement avec cette narrativité, car les cinq cordes de sémiotisation se recoupent, mais néanmoins prioritairement avec elle.

(94) Ainsi, dans une telle optique, le théâtre de Ionesco installe une distance sémiotique forte à l'égard de la grandiloquence et de la bonhomie de la théâtralité gaulliste d'après-guerre. En l'occurrence, il la contredit magistralement. 
Jean-Marie Privat sur Madame Bovary au milieu des années quatre-vingt-dix. Si les propos d'introduction comportent des formules devenues presque illisibles ${ }^{(95)}$, la lecture, elle, est très novatrice à la fois sur le plan de la démarche théorique et sur le plan de l'interprétation du roman. Privat s'oppose au préjugé voulant que le texte littéraire serait « un et indivisible ». Toute œuvre est au contraire culturellement métissée, hétérogène ${ }^{(96)}$. Non seulement elle fait fond sur la symbolisation culturelle des choses quotidiennes et de la vie ordinaire des gens, mais elle intériorise cette base culturelle et entreprend à son égard un travail de déplacement, de prospection, de dépense et de reconfiguration. C'est ce travail qu'il s'agit de décrire, d'interpréter et de comprendre dans le but de mettre en évidence le sens complexe qu'il donne au vivre-ensemble. Tandis qu'il pratique un doute méthodique à l'endroit de tout classocentrisme et qu'il adapte à l'étude de la littérature le mouvement poussant l'ethnologie contemporaine à migrer de l'exotique vers « l'endotique ${ }^{(97)}$, Privat remet en cause la fracture - particulièrement ouverte en France - entre culture populaire et culture savante et privilégie dans son essai l'examen de la reprise/transformation par le grand roman de Flaubert, auteur peu suspect de pactisation avec le populisme s'il en est, d'éléments issus du tuf symbolique associé à la vie paysanne ou provinciale et au folklore. C'est en partant du mot-valise « Charbovari » et de ses liens directs et indirects noués avec le mot « charivari» dès l'incipit du texte que la lecture prend son envol. L' « hypothèse charivarique »-elle mène en quelque sorte à définir le charivaresque - consiste à poser puis à démontrer que le roman flaubertien déplie au maximum le signe « charivari » et ouvre la configuration symbolique de la coutume du charivari à maintes possibilités narratives, sémiotiques et structurales. Dans la démarche ethnocriticienne, le repérage des éléments ethnographiques n'a d'efficience que dans la mesure où il conduit à la mise en valeur du «travail de signifiance que le texte opère ${ }^{(98)}$. » Ainsi, au prisme de la constellation de dénotations et de formulations escortant le charivari, sont successivement examinés les passages des traits de langue régionaux à la langue du roman, des façons de nommer les lieux et les gens à l'onomastique et à la topologie du texte, des représentations de la vie sociale villageoise ou provinciale à leur saisie en prose, de proverbes et de chansons populaires à leur insertion dans la logique narrative, des types pittoresques (le pharmacien de campagne) aux personnages fictifs (M. Homais). Dans la même logique, les aspects fondamentaux du charivari que sont le bruit, le débridé collectif, la violence symbolique ${ }^{(99)}$ sont repris et détournés par un roman tout entier consacré à décrire la socialisation de force d'Emma. Ce parcours, qui transpose l'affrontement folklorique séculaire de Carnaval contre Carême, transforme pour le meilleur et surtout pour le pire Emma Rouault en Madame Bovary, laquelle meurt de province, pourrais-je dire, en laissant résonner le titre et le sous-titre originels du roman. Le suicide d'Emma équivaut à une mort sacrificielle, refondant une société qui la préfère à tout dérangement de sa quiétude et de ses habitudes. L'analyse du Ventre de Paris d'Émile Zola proposée par Marie Scarpa adopte la même démarche, mais la précise et la présente de fa-

(95) Comme « la fraction dominée de la classe dominante » pour qualifier le lieu où seraient sociologiquement assemblés les intellectuels, les artistes, diverses corporations appariées et, sous leur nombre, des écrivains comme Flaubert.

(96) Cette hétérogénéité culturelle n’est évidemment pas un trait des seules littératures moderne et contemporaine.

(97) Il ne s'agit plus de chercher «l'autre» dans des cultures éloignées, mais de prendre en compte l'autre dans le familier, le lointain dans le proche, l'étrangeté dans le coutumier, etc.

(98) Jean-Marie Privat, Bovary Charivari. Essai d'ethno-critique, Paris, CNRS Éditions, 1994, 315 p., p. 31 .

(99) Le charivari est une pratique populaire « qui stigmatise avant tout, bruyamment et parfois très brutalement, très méchamment, tout individu qui enfreint, d'une manière ou d'une autre, le code dominant de la morale sexuelle ou conjugale traditionnelle » (ibid., p. 49). Le chahut de la classe de collège dans l'incipit est donc la métonymie du charivari global dont Emma est la victime en raison de son irrespect des règles du mariage. 
çon plus affermie : «Il va sans dire que l'ethnocritique ne se contente pas du simple repérage des faits ethnographiques, des "documents" et autres items folkloriques (ou folklorèmes, osons le terme) présents dans le texte littéraire savant; elle tend au contraire à en faire une véritable lecture, attentive à la manière dont ils en "travaillent" l'écriture même, soucieuse des logiques tant culturelles que textuelles qu'ils révèlent ${ }^{(100)}$. " Si l'intérêt primordial accordé " aux formes de culture subalterne » est réaffirmé, l'appui sur l'œuvre critique de Mikhäil Bakhtine, dont la convocation reste discrète dans Bovary Charivari, est souligné et étendu dans Le Carnaval des Halles puisqu'il est question de " continuer [son] héritage épistémologique». L'étude montre comment et combien le roman est habité par maintes représentations du monde sensible, de la corporéité et de l'existence au jour le jour, lesquelles tournent autour d'un rapport métaphorique coalisant le ventre et les Halles. L'irradiation de cette métaphore (par exemple via la mise en scène d'un lieu comme la charcuterie, la remotivation des topoï populaires de la lutte « des gras et des maigres » et du combat de Carnaval et de Carême, l'activation encyclopédique de l'intense complexe langagier lié à la culture du cochon) sur son ensemble permet au récit d'élaborer un tableau sophistiqué de la vie du quartier des Halles et de pointer la façon dont ses rites, usages et relations interindividuelles lui servent à encaisser les tensions socioculturelles et idéologiques conjoncturelles.

L'ouvrage suivant de Marie Scarpa est consacré au roman Le Rêve du même Zola, lequel raconte l'histoire d'une jeune fille passant d'abord de l'enfance à la puberté physiologique puis de l'adolescence au mariage (c'est-à-dire à l'âge adulte et au statut symbolique de «vraie femme ", car c'est l'homme qui, par le mariage, confère ce dernier). À Bakhtine s'adjoint une autre mobilisation théorique importante, déjà présente dans les deux essais qui viennent d'être commentés, mais cette fois davantage explicitée et plus opératoire : la théorie des rites de passage d'Arnold van Gennep, laquelle distingue en ces rites trois phases, l'une d'isolement, la seconde de marginalisation ou de « liminalité », la troisième d'agrégation ${ }^{(101)}$. Le Rêve est lu comme un récit d'initiation, ce dernier terme étant défini comme une " construction individuelle et sociale par l'apprentissage des différences de sexes, d'états [...] et de statuts ${ }^{(102)} »$ : dans ce processus entrent en jeu tout ce qui relève de la transmission (école, savoirs) et tout ce qui est de l'ordre de la culture environnementale (croyances, langages, pratiques, etc.). Forte de ces prémisses, la lecture du roman s'ouvre sur l'examen du métier de brodeuse qui est celui de l'héroïne, Angélique Rougon, et prend acte de ce que représentent les « travaux d'aiguille» dans l'éducation des jeunes filles au siècle de Victor Hugo, non pas en tant que fait brut ou statistique, mais en tant que pratique enrobée d'un imaginaire spécifique où évoluent des connotations, des valeurs, des marques de pouvoir et une fantasmatique conjuguant l'eros et l'anthropos. C'est de cette cristallisation imaginaire que le roman fait bombance. L'essai passe ensuite à l'examen des intertextes (Cendrillon, des légendes hagiographiques) et de l'interdiscours (formules langagières, traces d'oralité), puis à la série des rites déroulée par l'esthétique zolienne. Si Le Rêve du titre semble de prime abord désigner l'espoir stéréotypé de « rencontrer un prince charmant », la lecture ethnocritique révèle que le roman multiplie les visions oniriques concurrentes, quelquefois inquiétantes, et qu'il oppose l'espérance intérieure du sujet à une conception archaïsante du travail du rêve. Il laisse de la sorte voir combien le destin de la jeune femme est branché sur une " mémoire longue de la culture " que le texte à la fois prolonge et assaille. Mourant à la sortie de l'Église où vient d'avoir lieu son mariage avec le peintre-verrier Félicien, ma-

(100) Marie Scarpa, Le Carnaval des Halles. Une ethnocritique du Ventre de Paris d'Émile Zola, Paris, CNRS Éditions, 2000, $304 \mathrm{p}$.

(101) Arnold van Gennep, Les rites de passage, New York, Johnson Reprint, 1969 [1909], 288 p.

(102) Marie Scarpa, L'Éternelle jeune fille. Une ethnocritique du Rêve de Zola, Paris, Honoré Champion Éditeur, 2009, 269 p., p. 16. 
riage dont aucune des deux familles ne voulait, Angélique paie au prix fort l'écart commis à l'endroit des ritualisations sociales coutumières.

Quelques articles récents achèvent pour l'heure de décrire l'évolution de la démarche ethnocriticienne. Ouvrant son angle de réflexion bien au-delà du folklore et des symbolisations populaires sans pour autant les rejeter, elle s'intéresse de façon globale à « la pluralité culturelle constitutive des œuvres littéraires telle qu'elle peut se manifester dans la configuration d'univers symboliques plus ou moins hétérogènes et hybrides ${ }^{(103)}$ ». Elle envisage de produire une anthropologie des formes narratives et d'apporter une contribution à l'étude de leur historicité tout en caressant le souhait de faire donner ses principes directeurs sur l'analyse d'autres genres littéraires que le roman. L'héritage bakhtinien reste au cœur de sa démarche, mais ne se limite plus au grand œuvre sur Rabelais et le carnavalesque. L'ambition est d'accepter « toutes les conséquences de la polyphonie bakhtinienne du discours romanesque » (sc. plurilinguisme, plurivocalisme, hétérogénéités génériques et rhétoriques, etc.), de prendre en compte « les savoirs à l'œuvre » et de penser le processus d'acculturation réciproque unissant le texte et le lecteur. Au plan de la pratique méthodologique trois choses sont mises en évidence. Premièrement, l'attention portée aux éléments des textes qui se placent dans un entre-deux (entre deux répertoires axiologiques, entre deux registres culturels, entre deux temporalités), ainsi par exemple des personnages liminaires mis en texte par Zola ${ }^{(104)}$. Ensuite, l'adoption de la notion de «littératie ${ }^{(105)} »$, c'est-à-dire de la culture écrite, dont le fourmillement langagier, les intertextes et les interdiscours potentiels, les codes culturels et leurs jeux de permission/inter-diction constituent le matériel génétique du littéraire : la saisie de ce matériau et son traitement scriptural constituent la « logogenèse » à étudier ${ }^{(106)}$. En troisième lieu, le décèlement d' " embrayeurs culturels », définis comme des « points nodaux de l'œuvre réalisant la convergence du domaine référentiel extratextuel et du domaine littéraire au moyen de procédés d'écriture ${ }^{(107)}$. » Analysée par Guillaume Drouet, l'expression patoise " tso-maraude", accolée à Jean Valjean, en constitue un exemple dans Les Misérables : désignant un chat de maraude en son sens premier, elle signifie aussi marieur, berger, messager des morts, croquemitaine, au gré d'un rayonnement polysémique que le roman hugolien capte et transmute en autant de parcours narratifs et de ressorts sémiotiques possibles.

Lire le résumé qui précède ne peut que conduire à cette évidence : la sociocritique et l'ethnocritique sont plus que proches l'une de l'autre, et cette proximité est particulièrement forte avec l'École de Montréal (cf. supra). Le geste critique - étude interne des textes, éversion vers la genèse langagière, examen de l'interaction dynamique née de la dualité reprise/travail de l'écriture - est en son principe le même. Le recours aux idées fortes de Mikhaïl Bakhtine est commun aux ethnocriticiens et à maints sociocriticiens. La conception du texte et comme action sur le déjà-là et comme moyen d'invention de la « vie sociale » est la même. L'attention aux détails du texte, à ce qui paraît aller de soi mais qui ne le va pas du tout dès lors que se lit vraiment ce qui s'est écrit, est la même. Le souci d'étudier « les savoirs à l'œuvre » est identique ${ }^{(108)}$. Idem pour la mobilisation des res-

(103)Jean-Marie Privat et Marie Scarpa, "Présentation », dans Ethnocritique de la littérature, Romantisme, $\mathrm{n}^{\circ} 145$ ( $3^{\mathrm{e}}$ trimestre 2009), 174 p., pp. 3-9.

(104) Marie Scarpa, «Le personnage liminaire», ibid., pp. 25-35.

(105)Traduction de 1'anglais « litteracy». À propos de ce terme Marie Scarpa et Jean-Marie Privat renvoient à La logique de l'écriture de Jack Goody (Armand Colin, 1986), mais on n'omettra pas que le titre original de La culture du pauvre de Richard Hoggart est The Uses of Literacy. Aspects of workingclass life, with spécial reference to publications and entertainments (London, Chatto and Windus, 1957, 347 p.).

(106) Jean-Marie Privat, «Parler d'abondance. Logogenèses de la littérature », ibid.., pp. 79-95.

(107) Guillaume Drouet, «Les voies de l'ethnocritique », ibid.., pp. 11-23.

(108)Et est d'ailleurs central pour l'épistémocritique (Savoirs à l'œuvre est le titre d'un essai de Michel Pierssens, voir ici même, note 107). 
sources de la narratologie, de la théorie des genres et d'autres avenues de la « théorie littéraire ». Il y a bien là une réelle convergence et des rencontres à vouloir et à prévoir. Elles devront servir aux deux parties à revenir de façon critique sur les travaux des uns et des autres. Car s'il est des similarités fondamentales, il y a surtout là matières à discussion sur le plan de l'élaboration des concepts, de la conduite des lectures et de la formulation des hypothèses. Le préfixe « socio-" et le préfixe « ethno-» ne logent pas sous le même toit épistémologique, ainsi que le notait Marie Scarpa à la fin du Carnaval des Halles. La notion de «littératie » ou de «culture écrite » ne recoupe pas la « situation socio-linguistique» de Zima ou le concept de « discours social» de Marc Angenot. Quelles conséquences en tirer? Le mot «culture » est généralement absent des travaux sociocriticiens et, s'il y transite, n'est pris que dans un sens très vague. À l'inverse, le mot « discours » est rare ou allusif en ethnocritique. L'expression «culture populaire » est en déshérence chez les sociocriticiens : serait-ce que, en ne considérant que la « chose imprimée», ils ne prennent en compte que la «culture d'élite» ? L'orientation ethnologique et anthropologique des uns est-elle compatible avec l'inclinaison plus politique et philosophique des autres ? Le recours des ethnocriticiens à la théorie des rites de passage les conduit à postuler une homologie structurelle entre les ritualisations sociales et le genre romanesque. Un sociocriticien dirait plutôt que seule la tradition du roman de formation est concernée et que, de surcroît, les meilleurs de ses fleurons sont ceux qui indiquent que toute homologie de ce type est une illusion, à l'instar du Quichotte et du Père Goriot. Quant au roman contemporain (au sens très large), il semble naviguer sur des eaux bien plus troubles. $\mathrm{Ce}$ ne sont là que quelques notations rapides, auxquelles il faut espérer que les rencontres ici souhaitées donneront de l'étoffe dans l'avenir.

La saisie de l'éventail des discours que les textes prennent en écharpe débouche aussi de façon très intéressante sur des recherches plus ciblées. Rappelant que l'ère moderne est celle de la multiplication des pratiques et des professions et, par suite, de l'accroissement endémique des « discours spécialisés », Ursula et Jürgen Link remarquèrent très tôt que la littérature opéraient à leur égard un travail de recueillement et d'interfacialisation (dirait-on aujourd'hui). Ce travail mène tout particulièrement à la création de métaphores qui, par les connotations qu'elles activent et libèrent, agissent comme autant de passerelles symboliques : elles assurent ainsi une diffusion oblique des discours spécialisés vers l'ensemble de la formation sociale ${ }^{(109)}$. Les études qui, sous le chapiteau de l'épistémocritique, portent sur « la mise en texte des savoirs ${ }^{(110)}$ ", suivent les mêmes voies sans nécessairement aboutir aux mêmes destinations. Tout dépend bien sûr des textes considérés et des savoirs intégrés, car les sciences humaines ne passent pas en texte au prix des mêmes détournements, sélections, effacements, glissements que les sciences physiques ou les mathématiques. En plus de travaux théoriques, Michel Pierssens a apporté d'importantes contributions à ce domaine, arpentant les chemins de traverse reliant la littérature à la linguistique ou au savoir philosophique, découvrant du scientifique recyclé aussi bien chez Proust que chez des « fous littéraires ", s'occupant d'exonder de l'oubli des œuvres et des genres abandonnés par l'histoire littéraire à l'instar d'Ephraïm Michael et de la poésie scientifique ${ }^{(111)}$. Radicalement et forcément opposée à l'idée d'une autono-

(109) Ursula et Jürgen Link, "The Revolution and the System of Collective Symbols. Elements of a Grammar of Interdiscursive Events », dans Sociocriticism, 1 (1985), pp. 31-52, et Ursula Link-Heer, "La place de la littérature dans la gestion des sciences », dans Canadian Review of Comparative Literature, vol. 26, n 3-4 (2001), pp. 145-156.

(110) Je reprends ici le titre du collectif dirigé par Kazuhiro Matsuzawa et Gisèle Séginger (La mise en texte des savoirs, Strasbourg, Presses universitaires de Strasbourg, 2010, 352 p.).

(111) La Tour de babil, Paris, Minuit, 1976, 161 p. ; Lautréamont. Éthique à Maldoror, Lille, Presses universitaires de Lille, 1984, 213 p.; Savoirs à l'œuvre : essais d'épistémocritique, Lille, Presses universitaires de Lille, 1990, 185 p. ; [dir. Avec Franc Schuerewegen, et Ana Gonzàlez Salvador], Savoirs de Proust, Montréal, Paragraphes, vol. 26, 2005, 224 p. 
mie du littéraire que contredit le fait des textes, l'étude des relations intersémiotiques entre textes et savoirs ou entre littérature et sciences est un lieu très vivant de la recherche contemporaine. Il faudrait ici citer beaucoup de noms, dont celui de Jean-François Chassay, auteur de travaux remarqués sur des romans où la mobilisation du discours scientifique contribue à donner un sens généralement dysphorique, mais néanmoins équivoque, aux interférences entre les représentations du corps et celles de la ville, ainsi que sur la mise en scène et l'élaboration de la figure du savant dans des œuvres littéraires et cinématographiques ${ }^{(112)}$.

\section{La sociocritique qui se fait : voies d'avenir}

Les pages qui précèdent le prouvent : la sociocritique possède un fonds très considérable d'idées, d'élaborations conceptuelles, de propositions, d'approches, de résultats. Ce fonds est à entretenir sans doute, mais il n'y a pas ici de chaîne notionnelle à répéter et ânonner ad libitum pour servir un maître-penseur ou un mode d'emploi. Il faut une dernière fois le rappeler avant de placer le point final de cette synthèse par nature toute provisoire : la sociocritique est une perspective définissable par le geste critique qui la fonde, lequel fournit les linéaments d'une pratique de lecture des textes attentive à leur interaction avec la semiosis sociale qui les environne. À partir de cette base les chercheurs sont libres de travailler avec tous les outils d'analyse interne adéquats; ils sont aussi fortement invités à faire preuve d'imagination, sur tous les plans, et particulièrement sur le plan herméneutique ${ }^{(113)}$.

Il est toujours hasardeux de se livrer à la prospective dans le domaine des choses de l'esprit. Tâchant d'éviter les extrapolations abusives, un regard sur la sociocritique qui se fait met néanmoins à jour plusieurs chantiers prometteurs, en cours ou en voie d'établissement. Leur axe principal en est un de diversification salutaire. En raison des circonstances de son émergence, les corpus d'abord considérés par la sociocritique ont été des romans français du XIX ${ }^{\mathrm{e}}$ siècle (surtout) et du premier $\mathrm{XX}^{\mathrm{e}}$ siècle. Il n'y a aucune raison de ne pas continuer de ce côté, mais il est très important d'ouvrir à la lecture sociocriticienne tous les quartiers de l'immense ville des textes. Ce travail d'élargissement est en cours; il concerne les genres, les lieux, les temps.

Les études portant sur des genres autres que le roman doivent continuer à se multiplier. Le privilège accordé au genre romanesque vient de sa plus forte référentialité (apparente), de son penchant pour le «réalisme». Mais la sociocritique est conçue de manière à pouvoir aborder n'importe quelle écriture : j'irai jusqu'à dire que, plus le texte déroute les raccords référentiels, plus il la force à être bonne. Si elle a longtemps négligé la poésie, en dépit du fait que Walter Benjamin avait été en ce domaine un initiateur hors pair, c'était une erreur que plusieurs recherches ont déjà commencé à réparer. La contradiction du poème, citée ci-dessus, et le numéro "Sociocritique de la poésie » de la revue Études françaises ${ }^{(114)}$ ouvrirent des brèches en lesquelles se sont engagés plusieurs travaux dont, tout récemment, une thèse sur les représentations de la Première Guerre mondiale dans la poésie française (1914-1918) menée à terme par Olivier Parenteau, lequel

(112)Dérives de la fin. Sciences, corps et villes, Montréal, Le Quartanier, 2008, 224 p. ; Imaginer la science. Le savant et le laboratoire dans la fiction contemporaine, Montréal, Liber, 2003, 242 p. ; Si la science m'était contée. Des savants en littérature, Paris, Seuil, 2009, 303 p.

(113) Quitte à souligner que cette phrase n'engage que moi, je souhaite ardemment que les jeunes sociocriticiens soient caressés par ce vent de l'audace et de l'inventivité qui émane des livres d'un Pierre Bayard.

(114) Michel Biron et Pierre Popovic (dir.), Sociocritique de la poésie, Études françaises, vol. $27, \mathrm{n}^{\circ} 1$ (printemps 1991), $134 \mathrm{p}$. 
met en évidence la façon dont des recueils publiés par des auteurs comme Guillaume Apollinaire ou Jean Cocteau rompent avec les poncifs de la poésie patriotique pour s'efforcer de rejoindre la réalité de l'homme en guerre par des moyens scripturaux modernes ${ }^{(115)}$. Des études sociocritiques sur le théâtre ont aussi été conduites, par Claude Duchet, Pierre Laforgue, Xavier Bourdenet, etc. Il est essentiel de poursuivre dans cette voie sans omettre de prendre en compte les marques d'intergénéricité et que tout texte d'envergure refonde le genre où il s'inscrit.

La sociocritique s'est déployée avec bonheur sur des aires littéraires autres que l'Hexagone ${ }^{(116)}$. L'œuvre bakhtinienne, avec son attention aux plurilinguismes, aux polyphonies, aux formes hétérogènes, à l'interdiscursivité, avec son intérêt pour les rapports entre culture populaire et culture lettrée, ainsi que pour les relations entre écriture et oralité, a favorisé le développement de recherches sur des littératures où, en raison de circonstances historiques, des questions liées à ces enjeux théoriques se posaient. On citerait ici les travaux menés sur l'hétérolinguisme littéraire et sur une traductologie inspirée par la sociocritique à l'actif de critiques comme Rainier Grutman, Annie Brisset, Patrick Maurus ou Natalia Teplova ${ }^{(117)}$. Le domaine des recherches en littérature francophone et en littérature postcoloniale a particulièrement bénéficié de cet apport bakhtinien, ainsi que le montrent les essais de Christiane Ndiaye sur les littératures antillaises et africaines ou de Josias Semujanga et de Michael Rinn sur la littérature africaine et les récits du génocide au Rwanda ${ }^{(118)}$.

L'expansion vers des esthétiques et des œuvres négligées ou délibérément occultées par l'histoire littéraire - des textes pour la jeunesse, des romans édifiants, la " poésie scientifique », des hapax génériques, les prouesses des sogenannten « fous littéraires »est une excellente chose et pousse la lecture sociocriticienne à affronter à sa manière la question de l'oubli et cette « histoire des vaincus » à laquelle songeait Walter Benjamin. Il est d'ailleurs bon de rappeler que la sociocritique s'occupe des textes littéraires pour la raison nodale que ce sont eux qui offrent le plus de résistance à sa démarche, mais n'importe quel fragment de discours peut être considéré comme un texte ${ }^{(119)}$ : on peut faire l'étude sociocritique d'une chronique sportive, d'un faire-part de décès, d'un slogan politique, d'une chanson punk ${ }^{(120)}$, d'un fantasme d'époque ${ }^{(121)}$, d'une recette de cui-

(115)L'Honneur des poètes. Grande Guerre et modernité poétique (Apollinaire, Cocteau, Drieu La Rochelle, Éluard), Thèse (Ph.D), Université McGill, 2010, 455 p.

(116) Il faudrait citer ici beaucoup trop de travaux tels, entre cent, ceux portant sur les littératures hispaniques poursuivant dans la voie ouverte par Edmond Cros (Antonio Gomez Moriana, Daniel Meyran), sur la littérature américaine (Jean-François Chassay, Michel Nareau, Élaine Despré), sur la littérature belge (Rainier Grutman, Francis Mus), sur la littérature coréenne (Patrick Maurus), etc. etc.

(117) Rainier Grutman, Des langues qui résonnent: l'hétérolinguisme au XIX siècle québécois, Montréal, Fides, 1997, 222 p. ; Annie Brisset, Sociocritique de la traduction. Théâtre et altérité au Québec (1968-1978), Longueuil, Le Préambule, 1990, 374 p. ; Natalia Teplova, « Les migrations linguistiques de Vladimir Nabokov », dans TTR, vol. XVI, n² 2 (2003), pp. 137-154; Patrick Maurus, La mutation de la poésie coréenne moderne, ou les onomatopées fondatrices, Paris, L’Harmattan, 1999, 354 p. et Réflexions sur le poème en prose. Chu Yohan, Baudelaire, Paris, L'Harmattan, 2000, 206 p.

(118) Christiane Ndiaye, Danses de la parole. Études sur les littératures africaines et antillaises, Paris, L'Harmattan, 1997, 228 p. ; Josias Semujanga, Récits fondateurs du drame rwandais. Discours social, idéologies et stéréotypes, Paris, L’Harmattan, 1998, 256 p. ; Michael Rinn, Les récits du génocide. Sémiotique de l'indicible, Paris, Delachaux et Niestle, 1998, 288 p.

(119) L'idée n'est pas neuve et se trouve dans les premiers textes de Claude Duchet. Mais elle a mis du temps à se concrétiser.

(120) Sylvain David, « Un imaginaire de l'animosité. Le groupe punk Métal Urbain (1976-1978)», dans Marie-Hélène Larochelle (dir.), Invectives et violences verbales dans le discours littéraire, Québec, Presses de 1'Université Laval, 2007, pp. 91-110. Du même auteur, voir les travaux sur le nihilisme littéraire dont Cioran. Un hérö̈sme à rebours, Montréal, Presses de 1’Université de Montréal, coll. «Espace littéraire », 2006, 342 p.

(121)Claudia Bouliane, "Mourir dans la moyenne, à 130 à l'heure », dans François Provenzano et Sarah Sindaco (dir.), La Fabrique du Français moyen. Productions culturelles et imaginaire social dans la France gaullienne (1958-1981), Bruxelles, Le Cri/CIEL, 2009, pp. 65-77. 
sine ${ }^{(122)}$, et aussi celle d'un dessin, d'une photo, d'un tableau, d'une symphonie, d'un film à condition de prendre les outils analytiques ad hoc. En clair, tout dispositif sémiotique peut être l'objet d'une sociocritique, ce dont il dérive latéralement que des corpus composés, réunis dans une optique transmédiale, peuvent aussi donner lieu à des recherches fécondes ${ }^{(123)}$.

Les travaux contemporains jettent leurs lignes vers toutes les époques et ne privilégient plus le siècle de Balzac et de Victor Hugo. Les concepts doivent alors être travaillés et adaptés sous peine de produire des anachronismes. Les recherches de Francis Gingras sur le roman médiéval ouvrent à cet égard des pistes extrêmement fécondes, et qui ne concernent pas seulement les études médiévales, loin de là ${ }^{(124)}$. Nombre de thèses récemment déposées ou en cours abordent avec audace et perspicacité le roman contemporain et ses prolifiques altérités ${ }^{(125)}$. La littérature d'Ancien Régime n'est pas en reste, ainsi que l'attestent les travaux de Geneviève Lafrance, Joël Castonguay ou Geneviève Boucher ${ }^{(126)}$. En ces domaines, s'il importe de continuer à faire des monographies, il est bon aussi de prendre des corpus nombreux et intergénériques à bras-le-corps, sans rien concéder sur l'exigence d'analyse interne attentive des textes.

L'exemple magistral de Pierre V. Zima doit inspirer de plus en plus de recherches visant une internationalisation des corpus. Qui voudrait étudier le recyclage critique du discours consumériste dans la littérature des trente dernières années aurait tout intérêt à se donner une palette de romans où cohabiteraient entre autres et par exemple Americana de Don DeLillo, La carte et le territoire de Michel Houellebecq et Que notre règne arrive de Jérôme G. Ballard.

La sociocritique n'échappera pas à l' informatic turn. Si on ne voit pas très bien comment un logiciel pourrait faire de la sociosémiotique appliquée, il est cependant sûr que l'informatique est et sera d'un appoint important pour la collation des informations et des indices. Je ne pense pas seulement au fait de pouvoir voyager dans de larges corpus comme il est possible de le faire avec Frantext par exemple, mais à l'emploi des logiciels de lexicométrie qui ont remplacé les anciens « Dictionnaire de [La Comédie humaine], de [Proust], du [symbolisme] » fabriqués à la main au prix d'un effrayant travail de Romaine. Des outils tels Occurrences, Satorbase, Tropes, Weblex ou Alceste, même s'ils servent avant tout la linguistique textuelle et l'analyse du discours et si leur usage cultive abondamment l'illusion quantitative ${ }^{(127)}$, peuvent être utiles s'ils sont maniés avec précaution. Ils pourront notamment permettre de faciliter la cueillette de données dans l'étude d'un sociogramme. Au-delà de cette cueillette cependant, tout reste à faire, et surtout à lire.

Dès que le mandat qu'elle s'assigne est clair et respecté, la lecture sociocritique des textes peut évidemment se donner toutes les disciplines secondaires qu'elle veut et les

(122) Pierre Popovic, «La soupe aux choux : ingrédients et méthode », dans Études françaises, vol. 41, $\mathrm{n}^{\circ} 3$ (2005), pp. 41-61.

(123) Par exemple, l'analyse de la peinture officielle du second Empire peut entrer en parfaite résonnance avec celle des textes poétiques de Paulin Gagne.

(124)Érotisme et merveilles dans le récit français des XII et XIII ${ }^{e}$ siècles, Paris, Champion, 2002, $524 \mathrm{p}$.

(125) On verra entre autres les travaux de Mélanie Lamarre (sur Antoine Volodine et Olivier Rolin), Vicky Pelletier (sur J.G. Ballard), Djemaa Maazouzi (sur les représentations de la guerre d'Algérie), Émilie Brière (sur le motif de l'enfance violentée dans le roman actuel), etc.

(126)Joël Castonguay-Bélanger, Les écarts de l'imagination. Pratiques et représentations de la science dans le roman au tournant des Lumières, Montréal, PUM, 2008, 365 p. ; Geneviève Lafrance, Qui perd Gagne. Imaginaire du don et Révolution française, Montréal, PUM, 2008, 357 p. ; Geneviève Boucher, Histoire, Révolution et esthétique. Le temps et ses représentations dans le Tableau de Paris et le Nouveau Paris de Louis Sébastien Mercier, Montréal et Paris, Université de Montréal et Université de Paris IV-Sorbonne, thèse de doctorat en cotutelle, 2009, 2 vol., 575 p. [en voie de publication].

(127) L'idée que le haut degré de récurrence d'un lexème dans un texte littéraire soit la preuve de son importance sémiotique est un leurre issu de la confusion entre indice et valeur. 
discuter : elle trouve sans nul doute dans les sciences humaines en général des questions qui l'intéressent et qu' elle repose à sa guise à la littérature, mais les sciences physiques et celles dites « dures » peuvent elles aussi être inspirantes si le corpus l'exige ${ }^{(128)}$. Nombre d'approches critiques sont susceptibles de nourrir sa réflexion, dont au premier chef l'anthropologie littéraire ${ }^{(129)}$ et une partie des études intermédiales ${ }^{(130)}$. Des rencontres « interdisciplinaires » sont évidemment souhaitables, non qu'il faille faire " dialoguer» les " disciplines " les unes et les autres dans une sorte de moussaka pseudo-théorique mollement consensuelle, mais dans le but tout contraire de favoriser la confrontation des résultats et la discussion véritable, seule façon de conduire vers des affinements des lectures et des méthodes. Qu'elle soit sollicitée par la philosophie ou par une histoire culturelle qui fait quelquefois appel à elle ${ }^{(131)}$, elle y répondra dans l'esprit d'un " pluralisme fort [qui] expose les différences, [...] les mesure et les interroge, les traverse comme un incessant problème, comme un brouillage ou un désordre à assumer et à surmonter autrement que par des appels à l'unité, à l'identité et au recentrement ${ }^{(132)}$. »

C'est dans cette optique d'un pluralisme fort qu'il faut affirmer la nécessité de la sociocritique et la comprendre en regard du devenir actuel des études littéraires. C'est peu dire que de souligner que celles-ci ont aujourd'hui bien du mérite de subsister puisque la rumeur académique annonce périodiquement leur mort prochaine. Successivement déclarée mourantes par les sciences de la communication, par diverses sociologies, par le linguistic puis le cultural turn, ces études littéraires ont aussi en face d'elles un économisme néo-libéral et/ou un conservatisme vertueux (de gauche comme de droite), dont sont respectivement typiques le mépris pour ce qui ne rapporte pas « du cash » et la tradition de haine pour tout ce qui dit que nous ne vivons pas dans un monde immuable et magnifique. Qu'on ne s'y trompe pas : ces appels multipliés à la mort visent une création artistique qui, en démocratie, est l'une des formes capitales de la liberté d'expression. Parce qu'elle a rompu dès sa fondation avec les conceptions statique et sclérosantes de la critique de goût, parce qu'elle a refusé la démagogie du relativisme sociologique ou communicationnel des trente dernières années, parce qu'elle est à l'affût du dissident sociosémiotique, la sociocritique est à mon sens essentielle, et pas seulement aux études de lettres. À titre d'enseignant, je l'ai vérifié maintes et maintes fois, et dans plusieurs pays : partout où elle s'enseigne vraiment, la sociocritique attire de jeunes chercheurs. La raison en est qu' elle est capable de leur offrir une réelle défense de la littérature, à la fois respectueuse du travail spécifique opéré par le saut esthétique dans l'étrangeté qu'elle réalise et soucieuse de montrer qu'elle dit toujours quelque chose du monde pour peu qu'on veuille bien la regarder pour ce qu'elle est, de la fiction, du théâtre, du roman, un poème, c'est-à-dire une intervention individuée sur les mots qui convoient le cours des choses.

(128)Ce que montra Jean-François Chassay dans Le jeu des cö̈ncidences dans La vie mode d'emploi de Georges Perec, Lassalle, Hurtubise HMH, 1992, 172 p.

(129) On verra les travaux d'Alain Montandon, de Philippe Sabot, de Jean et Raphaël Molino, de Michel Fournier, etc.

(130) Notamment en raison de la présence dans maints textes littéraires de reproductions de peintures et de photographies (qu'on pense à nombre de recueils de poésie, à des œuvres de W. G. Sebald, de Claire Legendre, etc.).

(131)François Chaubet, " Histoire et culture à Pontigny-Cerisy », dans L'histoire culturelle du contemporain, Paris, Nouveau Monde Éditions, 2005, 436 p.

(132) Pierre Nepveu, L'Écologie du réel. Mort et naissance de la littérature québécoise contemporaine, Montréal, Boréal, 1988, 243 p., p. 215. 